\title{
L'encadrement communautaire de la concurrence en ZONE de l'Union Economique Monétaire Ouest Africaine (UEMOA)
}

\author{
Camara Mohamed ${ }^{1}$ \\ ${ }^{I}$ (Doctorant en droit public et sciences politiques au Centre des Etudes Doctorales en Droit et Economie- \\ Agdal/Rabat, Université Mohamed V, Maroc)
}

\begin{abstract}
The West African Monetary Union (WAEMU) was Created by Treaty on January 1Oth 1994 in Dakar, one of which key objective is to strengthen the competitiveness of the economic and financial activities of its Member States within the framework of an open market, competitive and a legal environment Streamlined and harmonized. The WAEMU competition law is published with the aim of establishing a common market in light of the European Union's experience, which has consecrated competition as a fundamental pillar of the single market. The EU suggest (preconizes) decentralization to member states structures while the WAEMU is moving towards the centralization of the exclusive competencies of the commission.

The WAEMU competition law comprises of primary and secondary laws which prohibit and sanction anticompetitive agreements, abuse of dominant position and aid from Member States. The WAEMU Laws on competition does not define competition and gives a predominant role to the Community bodies, in particular the Commission and the Court of Justice in its implementation.There are some institutional inconsistencies, in particular the inefficiency and the lack of collaboration between the Community bodies and the national competition structures. There is then a need to redefine the institutional orientation of the WAEMU for greater efficiency.
\end{abstract}

Key words: Abuse of dominant position and aid of Member States, primary and secondary legislation, anticompetitive agreements, common market, WAEMU (West African Monetary Union).

Résumé : L'UEMOA crée par le traité du 10 janvier 1994 à Dakar dont l'un de ses objectifs est le renforcement de la compétitivité des activités économiques et financières des États membres dans le cadre d'un marché ouvert, concurrentiel et d'un environnement juridique rationalisé et harmonisé. La législation de la concurrence UEMOA est éditée dans la perspective d'établissement d'un marché commun à l'image de l'Union Européenne qui a consacrée la concurrence comme un pilier fondamental du marché unique. L'UE préconise la décentralisation aux structures nationales des Etats membres alors que l'UEMOA s'oriente vers la centralisation de la compétence exclusive à la commission.Le droit UEMOA de la concurrence est composé du droit primaire et du droit dérivé qui interdisent et sanctionnent les ententes anticoncurrentielles, abus de position dominante et les aides d'Etats membres. Ce droit présente une carence de non définition de la concurrence et donne un rôle prépondérant aux organes communautaires notamment la Commission et la Cour de justice dans sa mise en œuvre.Il faut noter certaines incohérences institutionnelles notamment l'inefficacité de collaboration entre les organes communautaires et le structures nationales de la concurrence. Il y a alors une nécessité de redéfinir l'orientation institutionnelle de l'UEMOA pour plus d'efficacité.

Mots clés : Abus de position dominante et les aides des Etats membres, droit primaire et droit dérivé, ententes anticoncurrentielles, marché commun, UEMOA (Union Economique Monétaire Ouest Africaine).

\section{INTRODUCTION}

L'Union Economique et Monétaire Ouest-Africaine (U.E.M.O.A.), créée par le Traité de Dakar du 10 janvier 1994, s'inscrit dans une perspective substantiellement différente des schémas d'intégration l'ayant précédée en Afrique Occidentale. Les signes irrécusables de cettenouvelle perspective s'affichent tant au niveau de la structure institutionnelle que du système juridique. Par l'architecture institutionnelle qu'il met en place et l'ordre juridique qu'il assied, le système institutionnel de l'Union s'inscrit dans une autre perspective que celle ayant tendu à réduire les systèmes d'intégration au modèle ordinaire de l'organisation internationale, à savoir l'optique supranationale.L'organisation participe de cette mouvance et son Traité constitutif traduit dans plusieurs de ses dispositions, ce souci d'affranchissement de l'action communautaire des prérogatives souveraines, par l'introduction d'une dose de supranationalité. De ce point de vue, la stratégie adoptée pour la promotion de l'intégration et de la coopération régionale dans le traité de l'UEMOA entérine la transformation de l'Union Monétaire Ouest Africaine (UMOA) en union économique et elle regroupe Huit Etats côtiers et 
sahéliens, liés par l'usage d'une monnaie commune, le FCFA et bénéficiant de traditions culturelles communes, composent l'UEMOA : le Bénin, le Burkina Faso, la Côte d'Ivoire, la Guinée-Bissau, le Mali, le Niger, le Sénégal et le Togo. L'UEMOA couvre une superficie de $3506126 \mathrm{~km} 2$ et compte 112 millions d'habitants. Le taux de croissance du PIB est constantet est de $7 \%$ en $2015 .{ }^{1}$

La rupture totale avec les schémas qui ont jusque-là eu cours et introduit, dans le débat sur l'intégration, un jeu d'options originales portées par deux idées forces : un dessein de «communautarisation » et une volonté de $\ll$ juridisation $»^{2}$

\section{Carte 1: La carte des Etats membres de l'UEMOA}

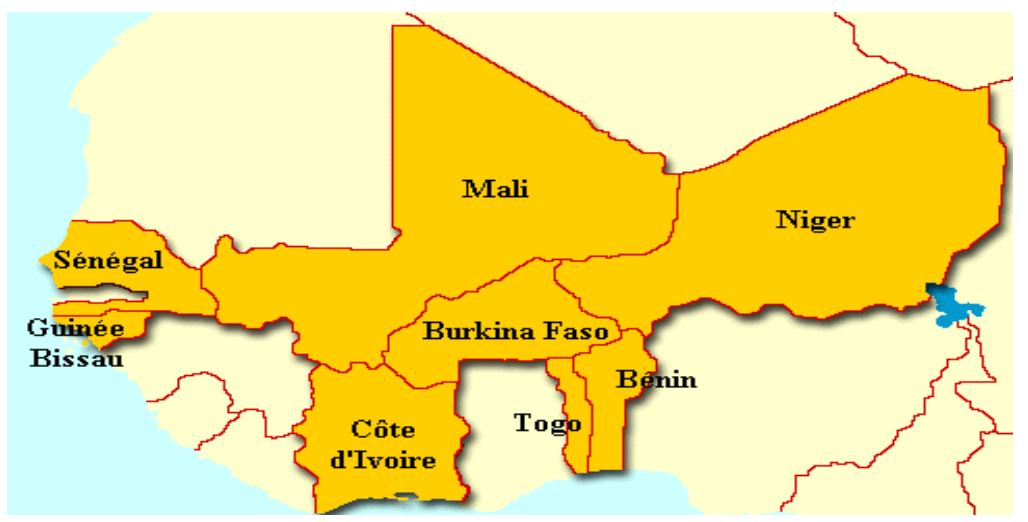

Source : www.izf.net

L'UEMOA est représentée par un logo symbolisant la croissance, l'union, la solidarité et la complémentarité entre les Etats côtiers et les Etats sahéliens. Elle a pour devise : « Huit pays, un destin commun ».Le logo représente deux formes dynamiques et elliptiques qui, malgré leurs spécificités, s'entremêlent pour exprimer deux notions fortes: la solidarité et la complémentarité des Etats et des peuples de l'Union.

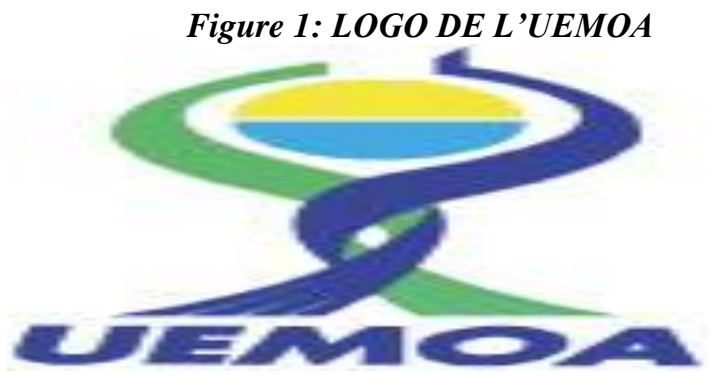

Source : Le site de l’UEMOA : $\underline{\text { www.uemoa.int }}$

Le Traité fondateur de l'Union Economique Monétaire Ouest Africaine (UEMOA) se fixe un certain nombre d'objectifs fondamentaux dont en particulier le renforcement de la compétitivité des activités économiques et financières de ses États membres dans le cadre d'un marché ouvert et concurrentiel et d'un environnement juridique rationalisé et harmonisé. Cet objectif est poursuivi en relation avec la création d'un marché commun basé entre autres sur la libre circulation des personnes, des biens, des services et des capitaux entre les États membres et sur l'institution d'un tarif extérieur commun (TEC) et d'une politique commerciale commune dans les rapports avec les pays tiers .

Ainsi, la concurrence apparaît d'entrée de jeu comme l'un des principes clés du processus d'intégration enclenché par l'UEMOA. Dans la suite logique des objectifs proclamés, ledit Traité indique également que, pour la mise en place du marché commun, l'Union œuvre pour l'institution de règles communes de concurrence applicables aux entreprises publiques et privées ainsi qu'aux aides publiques.Ces règles de concurrence retiendront l'attention, en raison notamment de la place capitale qu'elles occupent dans le schéma d'intégration mis en chantier par l'UEMOA et du grand intérêt qu'elles revêtent en cette époque de mondialisation et de libéralisation où la survie des acteurs économiques dépend en grande partie de leur capacité à résister à la concurrence. Dans le cadre de l'Union Européenne, le droit de la concurrence est plus qu'un objectif, c'est un pilier fondamental de la mise en place du marché unique. Ce rôle central dans la construction communautaire a conduit, en doctrine, à l'analyser en tant que « droit constitutionnel substantiel de la communauté européenne ». 
Poursuivant les mêmes objectifs, le législateur UEMOA s'est doté, à l'instar de son homologue européen, de textes de droit dérivé en vigueur depuis le 1er janvier 2003.

Les articles 88, 89 et 90 du traité posent les principes de base du droit communautaire de la concurrence par l'interdiction des ententes, des abus de position dominante et des aides d'État. Ils font de la Commission l'organe central de la mise en œuvre de règles communautaires par la compétence exclusive qui lui est reconnue.Si l'UE a choisi de décentraliser très largement l'application du droit communautaire de la concurrence en attribuant un réel pouvoir de décision aux juridictions nationales, le législateur UEMOA semble s'orienter vers une centralisation avec la reconnaissance d'une compétence exclusive de la Commission pour l'application des règles. Cette compétence exclusive de la Commission est cohérente avec celle reconnue par la Cour de justice UEMOA à l'Union pour légiférer pour tout ce qui est entente, abus de position dominante et aide d'État. En effet, dans son avis $\mathrm{n}^{\circ} 003 / 2000$, la Cour de justice de l'UEMOA, par une interprétation discutable des articles 88, 89 et 90 du traité de l'UEMOA, a conclu à une compétence exclusive de l'Union pour légiférer pour tout ce qui est entente, abus de position dominante et aide d'État. Les États membres n'ont dès lors de compétence législative que pour les domaines non couverts par le droit communautaire.

Si l'intégration économique et le contrôle de la libre concurrence au niveau régional ont le potentiel de catalyser le développement économique, l'expérience a fini de démontrer que, pour différentes raisons, leur mise en œuvre pose encore des défis. C'est à l'aune de ces défis majeurs (national et international) que plusieurs questions peuvent être posées.La question fondamentale que pose l'existence du droit de la concurrence de l'UEMOA est avant tout celle de la connaissance de son contenu exact. Dans cette optique, une première interrogation consiste à se demander quelles sont les règles de fond édictées par l'UEMOA pour garantir la libre concurrence et plus précisément quelles sont les interdictions et les obligations imposées aux différents protagonistes de la lutte concurrentielle ? Une seconde interrogation amène à se demander comment s'opère la mise en œuvre des règles de fond ou plus précisément quels sont les mécanismes prévus pour l'application de ces règles, qu'il s'agisse des organes chargés de cette application ou des procédures et sanctions destinées à garantir le respect de ces règles, et quels sont les éventuels rapports ou problèmes de compatibilité entre le droit de la concurrence de l'UEMOA et les autres droits de la concurrence applicables ou ayant vocation à s'appliquer dans l'espace UEMOA, que ce soit les droits nationaux ou les droits émanant d'autres organisations internationales ou supranationales ? Une autre question se pose sur l'efficacité et l'effectivité de la compétence exclusive accordée à l'Union notamment par le biais de la commission?

\section{ANALYSE DU CADRE JURIDIQUE DE LA CONCURRENCE ET DE SA MISE EN OEURE 'DANS L'ESPACE UEMOA}

Afin d'atteindre efficacement l'un des objectifs majeurs de l'UEMOA notamment de construire un espace économique harmonisé et concurrentiel, il a été adopté une « législation communautaire sur la concurrence» composée de Règlements et de Directives.

L'UEMOA recherche dans ce droit de la concurrence, à protéger le consommateur, à lutter contre l'inflation et à promouvoir la compétitivité internationale. De ce point de vue, il apparaît entre autres que le jeu normal de la concurrence entre les opérateurs économiques a un double avantage pour les pays africains : la satisfaction optimale des consommateurs et le dynamisme qu'elle influe à l'économie.

\section{1 - Le cadre juridique et sa mise en ouvre}

Pour atteindre efficacement l'objectif de renforcement de la compétitivité internationale des activités économiques et financières des Etats membres de l'UEMOA dans le cadre d'un marché ouvert et concurrentiel en vue de la réalisation du marché commun et face à l'existence ou à la menace permanente des pratiques portant atteinte à la concurrence, il s'est avéré nécessaire d'élaborer et de mettre en œuvre un ensemble de règles régulant ou disciplinant la concurrence. En d'autres termes, un droit de la concurrence applicable aux états membres. Pour que ce cadre juridique soit bénéfique à la concurrence, il faut un cadre institutionnel cohérent de manière à permettre un contrôle efficace tant des marchés nationaux que du marché commun, tout en garantissant une certaine harmonisation dans la mise en œuvre afin d'éviter les risques de renationalisation.

\section{1- La législation communautaire de la concurrence UEMOA et la grande contribution des actes dérivés}

Le droit communautaire de l'UEMOA sur la concurrence devait normalement comporter dès le démarrage du processus d'intégration deux grands volets, à savoir, d'une part, le droit primaire englobant les principes et les règles posés par le Traité constitutif de l'UEMOA du 10 janvier 1994, à travers précisément ses articles 76, 83, 88 à 90, et, d'autre part, le droit dérivé comprenant les règlements devant être pris par le Conseil des ministres de l'UEMOA dès l'entrée en vigueur du Traité, afin de faciliter l'application et de préciser des dispositions dudit Traité ${ }^{3}$.

Si ledit Traité est effectivement entré en vigueur très rapidement dès le 1er août 1994, il a fallu attendre le 23 mai 2002, soit plus de sept ans après le démarrage du processus d'intégration en question, pour assister à 
l'édiction du droit dérivé en matière de concurrence sous forme de règlements et de directives du Conseil des ministres de l'UEMOA. Ainsi, le droit communautaire de la concurrence issu de l'UEMOA comprend à l'heure actuelle aussi bien du droit primaire que du droit dérivé.

Le cadre juridique de la concurrence UEMOA, se compose du Traité et des normes dérivés. Il paraît important d'évoquer tout d'abord le mutisme du système normatif de l'Union sur la définition de la notion même de la concurrence.

\subsection{1- La carence de définition de la concurrence par le corpus normatif de l'UEMOA}

Les textes de l'UEMOA ne définissent pas directement les notions de concurrence, de politiques de la concurrence et, subséquemment, les concepts de pratiques portant atteinte à la concurrence. Cela pourrait poser des problèmes d'interprétation de tous ces concepts et de cerner précisément et de manière indiscutable leurs teneurs. Malgré l'absence de définitions directes, il ressort que « conformément à l'option libérale prise par l'UEMOA, le concept de concurrence repose sur le principe de liberté laissée à chacun des opérateurs économiques, la possibilité de produire, de vendre ce qu'il veut, aux conditions qu'il choisit. ». ${ }^{4}$ Cette conception et les instruments mis en place s'inscrivent dans la notion de concurrence praticable, imparfaite et réaliste ${ }^{5}$ par opposition à la conception d'une concurrence pure et parfaite ${ }^{6}$.

Ainsi, un marché où le jeu de la concurrence est libre est un marché où les entreprises, indépendantes les unes des autres, exerçant la même activité rivalisent pour attirer les consommateurs. Autrement dit, c'est un marché où chaque entreprise est soumise à la pression concurrentielle des autres.

Face à cette absence de définition, certaines définitions peuvent être proposées notamment celle qu'indique le Professeur Yves SERRA, « le droit de la concurrence comprend l'ensemble des dispositions juridiques qui régissent les rapports entre les professionnels dans leur activité concurrentielle pour réguler la concurrence $\gg^{7}$. La concurrence peut également être sommairement définie comme la compétition entre entreprises ou commerçants qui se disputent une clientèle ${ }^{8}$.

Selon le rapport ${ }^{9}$ cité plus haut, la raison de la non définition de la concurrence et les différents concepts connexes par les textes de l'UEMOA serait le fait que les Etats membres sont membres dans des organisations mondiales qui ont déjà défini ces notions telle que la CNUCED, et qu'elles sont universellement partagées.

Face à l'existence ou à la menace permanente des pratiques portant atteinte à la concurrence, il s'avère nécessaire d'élaborer et de mettre en œuvre un corps de règles les réprimant, un ensemble de règles régulant ou disciplinant la concurrence, ou, en d'autres termes, un droit de la concurrence s'inscrivant dans une politique de la concurrence. La politique de la concurrence quant à elle, peut être définie comme la gamme de mesures gouvernementales qui peuvent être employées pour promouvoir des structures et un comportement concurrentiel sur les marchés, y compris et sans exclure d'autres éléments, les lois de portée générale qui s'appliquent aux pratiques anticoncurrentielles des entreprises.

Si nous déplorons l'absence de définition claire de la notion de la concurrence par l'UEMOA, qu'est ce qui a été élaboré comme règle pour encadrer la concurrence dans l'espace de l'Union?

\subsection{2-Les règles communautaires de la concurrence comme instrument de création et de consolidation d'un véritable marché commun}

Le cadre juridique de la concurrence UEMOA, se compose du Traité et des normes dérivés.

Le Traité de l'UEMOA indique en son article 76, alinéa c, que, pour la mise en place du marché commun, l'Union œuvre pour l'institution de règles communes de concurrence applicables aux entreprises publiques et privées ainsi qu'aux aides publiques. Dans le paragraphe 4 de la section III du Traité («des règles de la concurrence»), trois articles concernent le droit de la concurrence et son application.

L'article 88 interdit:

- Les accords, associations et pratiques concertés entre entreprises, ayant pour objet ou pour effet de les restreindre ou de fausser le jeu de la concurrence à l'intérieur de l'Union;

- Toutes pratiques d'une ou plusieurs entreprises, assimilables à un abus de position dominante sur le marché commun ou dans une partie significative de celui-ci;

- Les aides publiques susceptibles de fausser la concurrence en favorisant certaines entreprises ou certaines productions.».

L'article 89 ajoute que le Conseil, statuant à la majorité des deux tiers (2/3) de ses membres et sur proposition de la Commission, arrête par voie de règlements, les dispositions utiles pour faciliter l'application des interdictions énoncées à l'article 88.

L'article 90 quant à lui, poursuit en indiquant que la Commission est chargée, sous le contrôle de la Cour de Justice, de l'application des règles de concurrence prescrites par les articles 88 et 89 . On peut résumer donc que les articles 88, 89 et 90 du traité posent les principes de base du droit communautaire de la 
concurrence par l'interdiction des actes anticoncurrentiels notamment les ententes anticoncurrentielles, des abus de position dominante et des aides d'État.

Pour ce qui est des ententes anticoncurrentielles, le Traité de l'UEMOA se borne pour l'essentiel à indiquer en son article 88, alinéa a, que les accords, les associations et les pratiques concertées entre entreprises sont interdits de plein droit, lorsque ceux-ci ont pour objet ou pour effet de restreindre ou de fausser le jeu de la concurrence à l'intérieur de l'Union, et à disposer en son article 89 que le Conseil des ministres arrête par voie de règlement dès l'entrée en vigueur du traité la procédure, les sanctions et les exceptions applicables à cette interdiction.Les dispositions susmentionnées consistent en réalité à poser le principe d'interdiction des ententes, à l'image notamment du droit européen et de différents droits nationaux de la concurrence ${ }^{10}$, puisque l'entente est classiquement définie comme un concours de volonté entre entreprises autonomes, tel un accord, une décision d'association, ou une pratique concertée, qui a pour objet ou pour effet de fausser ou d'entraver le jeu de la concurrence.Comme nous le laisse clairement apparaître la législation de l'UEMOA, la seule existence d'un concours de volonté entre entreprises autonomes ne suffit pas pour qu'il y ait entente prohibée. Il faut en plus que ce concours de volonté ait pour objet ou pour effet de restreindre ou de fausser la concurrence à l'intérieur de l'Union. L'évaluation de cet effet anticoncurrentiel, notamment par la Commission de l'UEMOA, doit être faite en utilisant le critère de la part de marché détenue par les parties à la pratique ${ }^{11}$. Plus précisément, la détermination de cette part de marché nécessite au préalable la définition précise du « marché en cause » qui apparaît comme le résultat de la combinaison entre «le marché de produits en cause » et le «marché géographique en cause ».En ce qui concerne l'interdiction des abus de position dominante, le Traité de l'UEMOA pose ici également, de façon très succincte le principe d'interdiction des abus de position dominante. A cet égard, l'article 88, alinéa b, dudit Traité dispose que sont interdits de plein droit toutes pratiques d'une ou de plusieurs entreprises, assimilables à un abus de position dominante sur le marché commun ou dans une partie significative de celui-ci. Ce sont surtout les dispositions du règlement $n^{\circ}$ 02/2002/CM/UEMOA et celles du règlement $\mathrm{n}^{\circ}$ 03/2002/CM/UEMOA qui viennent préciser le contenu de cette interdiction de principe des abus de position dominante ${ }^{12}$.

A travers de telles dispositions, la législation de l'UEMOA sur le droit de la concurrence met en exergue le fait que pour qu'il y ait abus de position dominante, il faut d'abord que l'entreprise en question soit en position dominante, et qu'ensuite cette entreprise exploite de façon abusive une telle position. Plus précisément, la position dominante est définie comme la situation où une entreprise a la capacité, sur le marché en cause, de se soustraire d'une concurrence effective, de s'affranchir des contraintes du marché, en y jouant un rôle directeur ${ }^{13}$.

De nombreux critères sont prévus pour déterminer l'existence d'une position dominante. Le critère le plus déterminant pour évaluer l'existence d'une telle position est la part de marché qu'occupe une entreprise sur le marché en cause. Cette part de marché se calcule en tenant compte des ventes réalisées par l'entreprise concernée et de celles réalisées par ses concurrents. Lorsque la part du marché ne suffit pas à elle seule pour établir l'existence d'une position dominante, les instances communautaires doivent recourir à des critères supplémentaires pour juger de celle-ci, tel :

- Le degré d'intégration verticale de l'entreprise, l'entrée.

- La puissance financière de l'entreprise ou du groupe auquel elle appartient, l'existence de barrières à

La seule existence de ces comportements abusifs ne suffit pas pour qu'il y ait abus de position dominante prohibé par le droit de la concurrence de l'UEMOA. Encore faut-il, en second lieu, que de tels comportements aient pour objet ou pour effet d'entraver de manière significative une concurrence effective à l'intérieur du marché commun.

Enfin, pour ce qui est de la prohibition des interventions étatiques, le régime communautaire en matière de régulation a engendré l'élaboration de règles qui s'appliquent directement aux entreprises, mais aussi aux Etats. Parmi lesquelles, l'interdiction des aides d'Etats dans la mesure où celles-ci affectent gravement les échanges. En ce sens, la définition large retenue par la jurisprudence, permet d'inclure dans la limitation des aides publiques, l'obligation de libéralisation et de transparence par rapport aux monopoles et entreprises publiques.

D'abord, sur la limitation des aides publiques, aux termes de l'article 88, alinéa c, du Traité de l'UEMOA, sont interdites de plein droit les aides publiques susceptibles de fausser la concurrence en favorisant certaines entreprises ou certaines productions, sous réserve d'exceptions limitées pouvant être prévues par le Conseil des ministres en vertu de l'article 89 du même traité. Ainsi est proclamée par ledit traité l'incompatibilité de la plupart des aides publiques avec le marché commun, puisque rares sont les aides susceptibles de favoriser toutes les entreprises ou toutes les productions ${ }^{14}$.

Une interdiction semblable existe également au niveau des règles de concurrence dans l'Union européenne. En effet, les articles 87 à 89 du Traité de la Communauté Européenne disposent de manière claire que la plupart des aides publiques sont incompatibles avec le marché commun. Poursuivant dans la même voie 
tracée par le Traité, le Règlement $n^{\circ}$ 04/2002/CM/UEMOA réitère une telle interdiction tout en apportant une précision sur son contenu et sa portée.

Au regard de ce dernier texte, la notion d'aide publique doit être entendue de la façon la plus large, aussi bien en ce qui concerne la forme de l'aide que la personne publique qui fournit l'aide. Ainsi, il apparaît que constitue une aide publique toute mesure qui entraîne un coût direct ou indirect, ou une diminution des recettes pour l'État, ses démembrements ou pour tout organisme public ou privé que l'État institue ou désigne en vue de gérer l'aide, et confère ainsi un avantage sur certaines entreprises ou sur certaines productions.

Cependant, s'inspirant de son homologue de l'UE, l'UEMOA a consenti quelques dérogations à cette règle d'interdiction à travers l'article 89, alinéa $\mathrm{c}$, du Traité de Dakar et le règlement $\mathrm{n}^{\circ}$ 04/2002/CM/UEMOA. Ces dérogations peuvent être résumées de la manière suivante :

- Les aides à des activités de recherche menées par des entreprises ou par des établissements d'enseignement supérieur ou de recherche ayant passé des contrats avec des entreprises, si l'aide couvre au maximum $75 \%$ des coûts de la recherche industrielle ou $50 \%$ des coûts de l'activité de développement pré concurrentielle;

- Les aides visant à promouvoir l'adaptation d'installations existantes à de nouvelles prescriptions environnementales imposées par la législation et/ou la réglementation qui se traduisent pour les entreprises par des contraintes plus importantes et une charge financière plus lourde, à condition que cette aide soit une mesure ponctuelle, non récurrente, et soit limitée à $20 \%$ du coût de l'adaptation ;

- Et, enfin, les aides destinées à promouvoir la culture et la conservation du patrimoine, quand elles ne restreignent pas la concurrence dans une partie significative du marché commun.

La deuxième source de la réglementation de la concurrence au niveau de l'UEMOA est le droit dérivé. Les textes de droit dérivé du 23 mai 2002, sont constitués de trois règlements et de deux directives à savoir :

- Le règlement $n^{\circ}$ 02/2002/CM/UEMOA relatif aux pratiques anticoncurrentielles à l'intérieur de l'Union économique et monétaire ouest africaine ;

- Le règlement $n^{\circ}$ 03/2002/CM/UEMOA relatif aux procédures applicables aux ententes et aux abus de position dominante à l'intérieur de l'Union économique et monétaire ouest africaine ;

- Le règlement $n^{\circ}$ 04/2002/CM/UEMOA relatif aux aides d'État à l'intérieur de l'Union économique et monétaire ouest africaine et aux modalités d'application de l'article 88 (c) du Traité ;

- La directive $n^{\circ} 01 / 2002 / C M / U E M O A$ relative à la transparence des relations financières d'une part entre les États membres et les entreprises publiques, et d'autre part entre les États membres et les organisations internationales ou étrangères ;

- Et, enfin, la directive $n^{\circ}$ 02/2002/CM/UEMOA relative à la coopération entre la Commission et les structures nationales de concurrence des États membres pour l'application des articles 88, 89 et 90 du Traité de l'UEMOA $^{15}$.

L'entrée en vigueur des trois règlements a eu lieu le 1er janvier 2003 tandis que celle des deux directives l'avait été le 1er juillet 2002, étant entendu qu'un délai de six mois jusqu'au 31 décembre 2002 avait été laissé aux États membres pour conformer leur législation interne à ces deux directives.

\section{2 - La mise en œuvre du droit UEMOA de la concurrence}

La mise en œuvre des règles de fond en toutes matières est la phase décisive qui permet de rendre effectives ces règles en recourant à un ensemble de mécanismes englobant aussi bien des institutions que des procédures et sanctions, tout en veillant à apporter des réponses adéquates aux écueils susceptibles d'entraver la bonne application de ces règles, au regard de la spécificité du contexte dans lequel se déroule cette mise en œuvre.Cette mise en œuvre est faible en raison de l'ignorance de la réglementation et de la procédure mais surtout du succès du règlement amiable informel des conflits relatifs à la concurrence. Toutefois, les organes internes de contrôle de la concurrence sont parfois saisis mais généralement par des sociétés commerciales. Ainsi, certaines affaires sont pendantes devant la Commission de la concurrence. De même, les juges connaissent rarement des litiges de la concurrence en raison de la lourdeur des procédures judiciaires et des connaissances limitées de la matière par ceux-ci. ${ }^{16}$

\subsection{1- Le rôle primordial des organes communautaires dans les procédures de mise en ouvre des règles de la concurrence UEMOA.}

Ce sont surtout les organes de régulation de la concurrence de l'UEMOA qui jouent un rôle primordial dans la mise en œuvre des règles de concurrence en suivant un certain nombre de procédures. Aussi, on peut d'abord identifier ces organes de régulation de la concurrence de l'UEMOA et leurs rôles respectifs avant d'examiner les procédures communautaires de mise en œuvre des règles de concurrence de cette organisation.

Aux termes du Traité de l'UEMOA et des textes de droit dérivé du 23 mai 2002, les principaux organes communautaires chargés de discipliner la concurrence au sein de l'UEMOA sont essentiellement le Conseil des ministres, la Commission, la Cour de justice et, dans une certaine mesure, le Comité consultatif de la 
concurrence.S'agissant du conseil des ministres, un rôle directeur lui a été attribué, ${ }^{17}$ c'est à dire une fonction de réglementation en matière de concurrence.

En effet, c'est le Conseil, statuant à la majorité des deux tiers (2/3) de ses membres et sur proposition de la Commission, qui arrête dès l'entrée en vigueur du Traité, par voie de règlements, les dispositions utiles pour faciliter l'application des interdictions énoncées à l'article 88 du Traité.

De même, il appartient au Conseil de fixer les règles à suivre par la Commission pour l'application des règles de concurrence, ainsi que les amendes et astreintes destinées à sanctionner les violations des interdictions énoncées.

Le Conseil peut aussi édicter des règles précisant les interdictions susmentionnées ou prévoyantes des exceptions à ces règles d'interdiction afin de tenir compte de situations spécifiques. C'est justement en s'appuyant sur de telles dispositions que le Conseil des ministres édictera les trois règlements et les deux directives du 23 mai 2002 sur la concurrence. A ce sujet, on peut à priori s'étonner de l'existence de deux directives parmi les textes de l'UEMOA sur le droit de la concurrence, alors même que l'article 89 du Traité de Dakar ne mentionne que des règlements, contrairement au Traité de Rome qui prévoit au profit du Conseil de semblables pouvoirs par voie de règlements ou de directives ${ }^{18}$. Pour bon nombre d'auteurs, le recours au règlement, par l'effet direct qui s'y rattache, conduit plus facilement à la pleine primauté du droit communautaire, puisqu'il permet d'éviter une certaine inertie des États que rend possible la directive qui, tout en liant les États membres quant aux buts à atteindre, leur laisse le choix des moyens pour y parvenir ${ }^{19}$. C'est aussi cette liberté de choix qui fait la souplesse de la directive, par opposition à la rigidité du règlement.

Toujours au niveau communautaire, au-delà du Conseil des ministres qui assure un rôle directeur en matière de concurrence, le rôle principal est confié à la Commission dans la mesure où celle-ci est chargée de l'application des règles de concurrence, en disposant, dans le cadre de cette mission, du pouvoir de prendre des décisions ${ }^{20}$.

A cet égard et en tant qu'une autorité administrative, a le droit d'être saisie, selon les cas, par voie de notification d'un accord, d'une décision, d'une pratique, d'une aide, par le biais d'une demande d'exemption, de dérogation ou d'attestation négative ${ }^{21}$, par le canal d'une plainte ${ }^{22}$ émanant d'une ou de plusieurs personnes intéressées, ou d'office ${ }^{23}$. Une fois saisie, elle a le pouvoir de faire un classement sans suite, d'ouvrir des enquêtes et de procéder à une instruction, en recourant à des auditions ${ }^{24}$, à des demandes de renseignements et à des vérifications ${ }^{25}$ et le pouvoir de prendre des décisions finales, sous forme par exemple d'autorisations ${ }^{26}$ ou d'exemptions ${ }^{27}$, d'injonctions et de mesures provisoires ${ }^{28}$, d'amendes et d'astreintes ${ }^{29}$. De surcroît, la Commission peut, par voie de règlements d'exécution, définir non seulement de nouvelles catégories d'aides publiques susceptibles d'être autorisées de plein droit, mais également des catégories d'ententes exemptées ${ }^{30}$.

La Commission de l'UEMOA a eu à connaître d'une pratique anticoncurrentielle qui intéresse directement le Sénégal : l'affaire RUFSAC. En application de la réglementation sur les aides d'Etat, le Département du marché régional, du commerce, de la concurrence et de la coopération de la Commission, dans son rapport d'étape du 14 avril 2008 sur l'affaire RUFSAC, demande à l'Etat du Sénégal de mettre un terme aux exonérations qu'il a accordées aux deux cimenteries importatrices d'emballages en papier que sont la SOCOCIM et les Ciments du Sahel. ${ }^{31}$

La Commission joue un rôle essentiel dans la conception et dans l'application du droit communautaire de la concurrence dans la mesure où, en tant que gardienne du Traité de l'Union, elle exerce une triple fonction dans le domaine de la concurrence : une fonction de réglementation; en second lieu, la Commission joue un rôle de définition de la politique de la concurrence de l'Union; en troisième lieu, elle est principalement chargée de la mise en œuvre du droit communautaire de la concurrence. L'action de la Commission se manifeste par des décisions, des avis ou des recommandations qu'elle adresse aux entreprises ou aux États membres. ${ }^{32}$

En plus de la Commission, un autre organe communautaire a un rôle essentiel à jouer en matière de concurrence : il s'agit de la Cour de justice de l'UEMOA qui doit surtout en ce domaine contrôler l'application des règles de concurrence par la Commission ${ }^{33}$.

Ainsi, la Cour de justice apprécie la légalité des décisions prises par la Commission en matière d'ententes et d'abus de position dominante, sur recours d'un Etat membre ou du Conseil, ou de toute personne physique ou morale intéressée ${ }^{34}$. De même, la Cour de justice statue, avec compétence de pleine juridiction, sur les recours intentés contre les décisions par lesquelles la Commission fixe une amende ou une astreinte, en ayant la possibilité de modifier ou d'annuler les décisions prises, de réduire ou d'augmenter le montant des amendes et des astreintes ou d'imposer des obligations particulières ${ }^{35}$.

En outre, relativement à la libéralisation des monopoles et entreprises publiques, la Cour de justice peut être saisie par la Commission lorsqu'un Etat membre ne se conforme pas à un avis ou une recommandation de cette dernière proposant des modifications à un projet de législation nationale susceptible d'affecter la concurrence à l'intérieur de l'Union ${ }^{36}$, par le recours en manquement.

A ces organes principaux prévus par le Traité pour réguler la concurrence, on peut également ajouter le Comité consultatif de la concurrence créé par l'article 28, paragraphe 28.3, du règlement $\mathrm{n}^{\circ}$ 
03/2002/CM/UEMOA du 23 mai 2002. Ce comité est composé de fonctionnaires compétents en matière de concurrence, à raison de deux représentants par État membre. Le Comité consultatif de la concurrence est consulté par la Commission de l'UEMOA pour avis, préalablement à toute décision en matière d'entente et $\mathrm{d}$ 'abus de position dominante ${ }^{37}$ et avant certaines décisions en matière d'aides publiques dont, en particulier, les décisions conditionnelles et les décisions négatives ${ }^{38}$.

Les différents organes de concurrence de l'UEMOA exercent leurs rôles respectifs à travers les procédures de mise en œuvre des règles de concurrence de cette organisation.

\subsection{2- Les procédures de mise en œuvre des règles de concurrence de l'UEMOA et les sanctions en cas d'infraction aux règles}

Ces procédures se déroulent essentiellement auprès de la Commission de l'UEMOA, de façon semblable à la situation ayant cours au niveau de l'Union européenne. Cette partie peut être scindée en deux notamment :

Dans un premier temps, il y a les procédures de mise en œuvre des règles de concurrence UEMOA. Dans la procédure non contradictoire, dès réception d'une notification ou d'une demande émanant d'une ou de plusieurs personnes intéressées ${ }^{39}$, la Commission publie une brève communication sur l'accord, la décision ou la pratique en question, l'objet de cette communication étant d'inviter les parties tierces à faire des observations sur ceux-ci. Dans les six mois à compter de la notification ou de la demande, la Commission peut décider d'octroyer une attestation négative ou une exemption individuelle, si aucun grief n'est retenu contre une des parties intéressées. Lorsque la Commission émet des doutes sur la compatibilité des accords, décisions et pratiques concertées avec le marché commun, elle peut alors décider d'initier la procédure contradictoire ${ }^{40}$.

La procédure contradictoire est initiée sur décision de la Commission de l'UEMOA avec communication de griefs, suite à une plainte ${ }^{41}$, une notification émanant d'une ou plusieurs personnes intéressées, ou de sa propre initiative, en vue de l'obtention d'une décision d'attestation négative, d'exemption individuelle, ou de condamnation pour infraction à l'interdiction des ententes et des abus de position dominante. La communication des griefs est faite par la Commission de l'UEMOA qui transmet par écrit, à chacune des entreprises et associations d'entreprises ou à leur mandataire commun, les griefs retenus contre elles, en leur laissant la possibilité effective de présenter leur défense par écrit ou oralement lors d'auditions ${ }^{42}$.

La demande d'attestation négative vise à faire constater par la Commission qu'il n'y a pas lieu pour elle d'intervenir à l'égard des pratiques mises en œuvre. Pour bénéficier d'une exemption individuelle ou d'une attestation négative, les entreprises sont tenues d'en faire la demande ou de notifier la pratique en cause au moyen du formulaire (N)3 qui fournit toute une série d'informations concernant les parties, les accords, le marché considéré et les raisons pour lesquelles la demande de la notification est présentée. Si aucune décision n'est prise au-delà de 12 mois, à compter de l'ouverture de la procédure contradictoire, ce silence des autorités communautaires équivaut à une décision implicite d'attestation négative ou d'exemption.

En matière d'aides publiques, les textes de l'UEMOA sur la concurrence envisagent quatre sortes de procédures : la procédure concernant les aides notifiées, la procédure en matière d'aides illégales, la procédure en cas d'application abusive d'une aide et enfin la procédure relative aux régimes d'aides existantes ${ }^{43}$.

La procédure en matière d'aides illégales est mise en branle lorsque la Commission a en sa possession des informations concernant une aide prétendue illégale. Pour clôturer la procédure, la Commission peut décider que l'État membre concerné doit prendre toutes les mesures nécessaires pour récupérer l'aide auprès de son bénéficiaire. La procédure en cas d'application abusive d'une aide se ramène à la possibilité pour la Commission d'ouvrir une procédure semblable notamment à celle relative aux aides illégales ${ }^{44}$.

En ce qui concerne la procédure en matière d'aides existantes, la Commission procède avec les États membres à l'examen permanent de ces régimes, avec la possibilité de faire des recommandations proposant l'adoption de mesures utiles ${ }^{45}$. Si l'État membre concerné ne se conforme pas à une décision conditionnelle ou à une décision négative ou à une injonction de suspension ou à une injonction de récupération ou à une décision de récupération ou aux arrêts de justice y afférents, la Commission peut, après avoir invité l'État membre à faire ses observations, prendre des mesures graduelles ${ }^{46}$.

Au-delà de ces procédures mentionnées ci-dessus que certains qualifient de gracieuses, il y a aussi les procédures contentieuses pour la mise en œuvre des règles de concurrence UEMOA.

En effet, le contentieux dans le droit de la concurrence de l'UEMOA obéit à deux règles fondamentales qui sont le respect du contradictoire et la transparence des procédures.

La procédure contradictoire est initiée sur décision de la Commission de l'UEMOA avec communication de griefs. Elle se fait suite à une plainte, une notification émanant d'une ou plusieurs personnes intéressées, ou de la propre initiative de la Commission.

La communication des griefs est faite par la Commission de l'UEMOA qui transmet par écrit, à chacune des entreprises et associations d'entreprises ou à leur mandataire commun, les griefs retenus contre elles, en leur laissant la possibilité effective de présenter leur défense par écrit ou oralement lors d'auditions. 
Pour mener ces enquêtes en vertu du règlement $n^{\circ}$ 03/2002/CM/UEMOA du23 mai 2002, la commission procède en règle générale de deux manières à savoir : une enquête sous la forme de demande de renseignements adressés aux entreprises et associations d'entreprises. Mais elles peuvent aussi consister en des vérifications sur place.

La procédure d'instruction devant la Commission revêt trois caractères : elle est contradictoire, elle garantit le respect des secrets d'affaires et enfin, elle est menée en étroite collaboration avec les États membres.

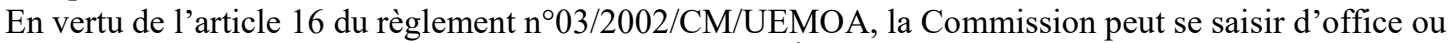
être saisie par un plaignant. Les plaignants peuvent être soit des États membres soit des personnes physiques ou morales.

La procédure contradictoire comporte deux phases :

- La phase écrite qui consiste à la communication de griefs, à l'accès au dossier et aux observations écrites des parties ;

- La phase orale, il est prévu qu'au aux termes de la communication de griefs, la Commission doit procéder à une audition des parties contre lesquelles elle a retenu des griefs en vue de l'adoption d'une décision.

Dans un second lieu, il y a les procédures pour les sanctions des pratiques anticoncurrentielles. La Commission de l'UEMOA qui est chargée de défendre l'ordre public économique, notamment dans sa dimension concurrentielle, veille à l'application effective de la règlementation sur la concurrence en disposant du pouvoir de prendre des sanctions.

Dans le domaine de la concurrence, les sanctions sont de deux ordres : les décisions constatant des infractions et les décisions infligeant des amendes ou des astreintes.

Les sanctions prévues contre les auteurs de pratiques anticoncurrentielles sont au nombre de trois :

- La nullité qui sanctionne les accords et décisions interdits ;

- Les amendes et les astreintes infligées aux entreprises coupables de pratiques anticoncurrentielles;

- Et les sanctions spécifiquement prononcées contre les Etats pour non-respect de décisions ou d'arrêts. ${ }^{47}$

A côté des sanctions à l'encontre des entreprises, il y a également des sanctions prévues à l'égard des Etats membres pour cause de pratiques anticoncurrentielles. En vertu de l'article 5 du Règlement $n^{\circ}$ 04/2002/CM/ UEMOA relatif aux aides d'Etat à l'intérieur de l'UEMOA et aux modalités d'application de l'article 88 (c) du Traité « tout projet d'octroi d'une aide nouvelle est notifiée à la Commission par l'Etat membre concerné ».Dans toutes les situations où la procédure aboutit à une décision négative concernant une aide illégale, la Commission décide que l'Etat membre concerné prenne toutes les mesures nécessaires pour récupérer l'aide auprès de son bénéficiaire : c'est la décision de récupération de l'article $16.1 \mathrm{du}$ ledit règlement.

\section{2- La problématique de la cohérence institutionnelle pour la mise en œuvre de concurrence UEMOA}

Ces passages seront réservés à l'analyse de l'architecture institutionnelle de mise en œuvre de la politique régionale de la concurrence UEMOA. Rappelons que l'UEMOA a choisi un système institutionnel fortement centralisé notamment au niveau de la commission. Cette forte centralisation à montrer certaines limites qu'il convient de présenter ici. D'ailleurs, la doctrine est très critique sur cette forte centralisation des compétences au niveau de l'UEMOA ${ }^{48}$.

\section{1- Les incohérences institutionnelles au niveau communautaire et national}

Au niveau communautaire, les questions consistent à savoir si la Commission de l'UEMOA a-t-elle les moyens de sa politique ? En d'autres termes, est-ce que la Commission de l'UEMOA a les capacités, les ressources matérielles et humaines et la flexibilité nécessaires pour mettre en œuvre efficacement le droit communautaire de la concurrence ? Les procédures communautaires garantissent-elles une intervention efficace de la Commission sur le marché ? Nous allons essayer de répondre à ces questions.

A titre de rappel, les services de la Commission de l'UEMOA sont organisés en départements ${ }^{49}$ qui s'articulent autour de la présidence de la Commission. Chaque département est dirigé par un commissaire. À leur tour, les départements sont éclatés en directions. C'est le département du marché régional, du commerce, de la concurrence et de la coopération ${ }^{50}$ qui coiffe la direction de la concurrence en charge de la mise en œuvre de la politique communautaire de la concurrence. C'est seulement en 2007 que la Direction de la concurrence a été créée. Avant cette date, le contentieux concurrentiel était traité au sein du département du marché régional, du commerce, de la concurrence et de la coopération. Aucune particularité n'était accordée au contentieux concurrentiel. Le département de la concurrence manquait de visibilité.

Par ailleurs, une certaine lourdeur administrative limite l'effectivité de l'action de la Direction de la concurrence. Comme dans toutes les administrations classiques, les actions de cette dernière doit avoir l'aval de sa hiérarchie. Avant toute vérification dans un État membre ou une entreprise, la Direction de la concurrence doit introduire une demande auprès du président qui juge de l'opportunité du déplacement avant de signer un ordre de mission. Ces contraintes procédurales internes limitent considérablement la flexibilité de la 
Commission dans la conduite des enquêtes. C'est également le président de la Commission qui signe tous les actes de procédure (demandes de renseignements, communications avec les parties, etc.).

En termes du personnel, là aussi, la direction de la concurrence a un déficit criant car il y a seulement 3 agents pour s'occuper de tout le contentieux communautaire en la matière. Certes, il est demandé aux structures nationales de la concurrence de fournir des agents lors des enquêtes menées par la direction, mais cet appui national, qui du reste est limité, ne comble pas le déficit en personnel de la Commission ${ }^{51}$.

Pour que la direction de la concurrence UEMOA soit efficace, il faut qu'elle soit dotée des moyens financiers suffisants et en personnels. Mais dans le cas de l'UEMOA, malgré que la commission dispose de moyens financiers suffisants, la direction de la concurrence ne bénéficie pas de la flexibilité nécessaire quant à leur utilisation. $^{52}$

\subsection{1 - Les incohérences au niveau national : la problématique de la collaboration des structures nationales de la concurrence}

En ce qui concerne les incohérences au niveau national notamment la problématique de la collaboration des structures nationales de la concurrence, il est attendu des réformes au niveau national et une collaboration effective des structures nationales de la concurrence. Dans les États membres, les réformes ne sont pas encore effectives et la collaboration des structures nationales de la concurrence avec la Commission n'est pas au rendez-vous.

Les incohérences au niveau national s'aperçoivent d'abord par l'ineffectivité des réformes institutionnelles au niveau des États membres. Une certaine léthargie dans le fonctionnement des structures nationales de la concurrence et un ralentissement de la dynamique législative nationale ont suivi l'entrée en vigueur du droit communautaire de la concurrence. En réalité, la léthargie des structures nationales de la concurrence est endémique et congénitale. En effet, avant l'entrée en vigueur du droit communautaire, elles avaient toute la latitude de mettre en œuvre leur politique nationale de concurrence. Toutefois, aucune d'entre elles n'a effectivement mis en œuvre son droit de la concurrence. Même une certaine doctrine soutient que l'entrée en vigueur du droit communautaire a entraîné un recul de la compétence nationale ${ }^{53}$. La direction de la concurrence de l'UEMOA avait des structures nationales de la concurrence notamment au Sénégal et en Côte d'Ivoire. Elles avaient toute la latitude de mettre en œuvre leur politique nationale de concurrence. Toutefois, aucune d'entre elles n'a effectivement mis en œuvre son droit de la concurrence.

Le cas de la Commission nationale de la concurrence de la Côte d'Ivoire est très intéressant à ce propos. Créée en 1992 avec la Loi sur la concurrence, la Commission a été opérationnelle jusqu'en 2002. Des moyens conséquents ont été mis à sa disposition et elle a eu à rendre pas moins de 30 Avis. C'est suite à une crise sociopolitique que son activité a été ralentie.Les droits nationaux de la concurrence ne sont pas encore mis en conformité avec le droit communautaire malgré l'obligation faite aux États membres de réformer leurs législations suite à l'entrée en vigueur de ce droit. En effet, aucun pays membre de l'UEMOA n'a à ce jour mis en conformité son droit national avec le droit communautaire. A cela s'ajoute l'inefficacité de la collaboration des structures nationales de la concurrence qui se manifeste par la faiblesse institutionnelle des structures nationales de la concurrence.

\subsection{2-L'ineffectivité de la collaboration des structures nationales de la concurrence}

Il y a quelques années, il a été évoqué un risque d'ineffectivité de la collaboration des structures nationales de la concurrence à la mise en œuvre du droit communautaire. Dépourvues de toute compétence de prise de décision, nous soutenions que les autorités nationales de la concurrence ne seraient pas enclines à collaborer effectivement avec la Commission. ${ }^{54}$ Cette résistance était prévisible. Les structures nationales de la concurrence n'exercent pas leur rôle de veille concurrentielle des marchés nationaux; les cas de transmission des plaintes à la Commission de l'UEMOA sont limités ; et dans certains cas, il y a une absence totale de collaboration lors des enquêtes ${ }^{55}$. À part l'autorité de la concurrence du Burkina Faso qui s'est montrée très coopérant avec celle-ci, les structures nationales de la concurrence ne sont en général pas enclines à collaborer avec la Commission. ${ }^{56}$

La faiblesse institutionnelle est l'une des raisons de cette ineffectivité de cette collaboration. Le système institutionnel communautaire s'appuie sur les structures nationales pour son effectivité même si ces dernières sont dépourvues de pouvoir de décision. A titre d'exemple, La Guinée-Bissau n'a pas de structure nationale de la concurrence, encore moins de droit de la concurrence. Théoriquement, cet appui sur les institutions nationales n'existe pas dans cet État.Il y a lieu de rappeler pour terminer, il y a les problèmes de la disponibilité des ressources humaines et matérielles de ces structures nationales de concurrence car celles-ci ne sont pas dotées de moyens suffisants et leur personnel qui manque souvent de qualification en droit de la concurrence. 


\section{2- La nécessité de redéfinir l'orientation institutionnelle de l'UEMOA pour plus d'effectivité}

Dans le cadre de l'UEMOA, la réflexion est déjà engagée sur un possible réforme du cadre institutionnel de mise en œuvre des règles communautaires de la concurrence. Une étude est commanditée dans ce sens par la Commission de l'UEMOA. Les autorités communautaires, après plusieurs années de mise en œuvre du droit communautaire, se sont rendu compte des blocages du système centralisé. La coopération des structures nationales de la concurrence avec la Commission n'est pas effective, ce qui limite considérablement l'effectivité du droit communautaire.

Les structures nationales de concurrence n'ayant jamais accepté la compétence exclusive de la commission, ont toujours réclamé une redéfinition des compétences entre la Commission et les structures nationales de la concurrence. La formule «tout ne peut pas se faire à Ouaga ${ }^{57}$ est devenue familière. Plus qu'un simple slogan, elle constitue un cri de résistance et exprime le besoin de réformer un système qui a montré ses limites. Mais dans quelle direction réformer?

Pour cela, il faut de prime abord, une décentralisation contrôlée, de la mise en œuvre du droit communautaire de la concurrence dans le cadre de l'UEMOA. Il faut ensuite, une redéfinition des compétences entre l'Union et les États membres dans le cadre de l'UEMOA qui peut s'opérer suivant deux principes : la centralisation des standards du droit matériel et la décentralisation de la prise de décision.

Il est utile de préciser que la centralisation des standards du droit substantiel ne doit pas nécessairement conduire à une disparition totale des législations nationales de la concurrence. En effet, il est crucial dans ce processus de reconnaître aux États membres la possibilité d'avoir des droits nationaux qui peuvent couvrir des domaines allant au-delà des pratiques anticoncurrentielles stricto sensu comme la concurrence déloyale et les pratiques restrictives de concurrence, qui, dans l'UEMOA, relèvent de la compétence des États membres. Reconnaître aux États la possibilité d'avoir des droits nationaux de la concurrence et encourager un certain dynamisme législatif au niveau national participent à la promotion de la culture de la concurrence dans les États membres.

En ce qui concerne le droit matériel, Il est concevable dans le cadre de l'UEMOA de maintenir la réglementation unifiée des pratiques anticoncurrentielles tout en continuant les réformes visant à mettre en conformité les droits nationaux avec le droit communautaire de la concurrence. Toutefois, il est important que les États membres développent leurs droits nationaux de la concurrence en droite ligne avec les standards communautaires.

\section{DE LA COMPETENCE EXCLUSIVE DE L'UEMOA EN MATIERE DE CONCURRENCE}

L'existence d'une réglementation de la concurrence UEMOA soulève la question du sort des réglementations nationales en la matière et sur leurs possibles cohabitations dans le même espace. Saisie par la commission de l'UEMOA, dans le cadre de l'élaboration du droit dérivé de la concurrence, sur l'interprétation à donner aux règles de l'article 88 quant à la coexistence de la législation communautaire et des législations nationales, la Cour de justice de l'UEMOA a retenu le principe de l'exclusivité du droit communautaire de la concurrence. Cette règle est explicitement énoncée par la Cour dans son avis $n^{\circ}$ 003/2000 du 27 juin $2000 .{ }^{58}$ Selon la Cour, la production et l'application des règles de la concurrence relèvent de la compétence exclusive des organes communautaires. Elle a, en cela, suivi la commission de l'UEMOA. S'inscrivant dans une vision d'un droit à vocation centralisatrice, la Cour énonce: «Ce principe exclusiviste ne permet pas aux Etats membres de légiférer de plein droit dans les matières de l'article 88 du traité .... Exception faite de prescriptions formelles des autorités communautaires les associant à l'exercice de cette compétence qui lui est dévolue ». Le juge communautaire, usant pleinement de la marge d'interprétation que lui reconnaît le traité, a fait une véritable œuvre législative en orientant de la sorte le droit communautaire. Les Etats membres se voient ainsi retirer tout pouvoir de réglementation dans le domaine réservé à l'Union. La différence avec le droit de la concurrence de la communauté économique et monétaire de l'Afrique centrale (CEMAC) et dans l'Union européenne est patente. ${ }^{59}$ Ces systèmes retiennent en effet la coexistence des deux ordres des textes en affirmant la primauté du droit communautaire. ${ }^{60}$ La règle est explicitée par la jurisprudence européenne et sa première expression remonte à l'arrêt Costa du 15 juillet $1964 .{ }^{61}$ L'exclusivité apparaît comme la pierre angulaire de toute la construction communautaire UEMOA de la concurrence, puisque dans l'élaboration du droit dérivé de la concurrence, la commission est appuyée sur l'avis de la Cour de justice.Ce principe peut susciter des interrogations. Une règle qui réduit considérablement la liberté des Etats membres ne peut être acceptée que si lorsque cette dernière est solidement justifiée. Dans quelle mesure l'affirmation de la compétence exclusive des organes de l'Union en droit de la concurrence est-elle fondée ? Est-elle souhaitable ?

La règle du monopole de l'ordre communautaire est une norme que peut sembler justifier l'objectif de construction d'un marché unique. En effet, lorsque l'on vise une réalité économique unique, le droit destiné à l'encadrer ne devrait -il pas être, lui aussi, unique ${ }^{62}$ ? 
A l'analyse, l'exclusivité est cependant introuvable. C'est en vain qu'on cherche un fondement à la compétence exclusive des organes de l'Union en droit de la concurrence dans l'UEMOA. Pour consacrer ledit principe, la Cour de justice s'est inscrite dans un cadre textuel très limité et est allé au-delà dans l'intention des rédacteurs du traité de Dakar.

\section{3. - Les fondements d'une compétence exclusive à l'UEMOA discutable}

Il s'agira ici d'essayer d'exposer le débat qui a eu lieu autour de la question de la compétence exclusive entre la commission et les experts des Etats membres. Cette exclusivité qui a un fondement jurisprudentiel qui donne un monopole à l'Union notamment le rôle prépondérant de la commission en la matière.

\section{1 - Le débat autour de la compétence exclusive de l'UEMOA}

Face à la coexistence des législations nationales et de la législation communautaire en matière de concurrence, deux camps ont commencé à s'affronter dès la phase d'élaboration des textes de l'UEMOA sur le droit de la concurrence, par exemple lors des travaux de « l'atelier sur le projet de législation communautaire de la concurrence à l'intérieur de l'Union » tenu au siège de la Commission de l'UEMOA, à Ouagadougou, du 10 au 14 avril $2000^{63}$.Pour le premier camp regroupant notamment les experts des pays membres de l'UEMOA, les législations nationales doivent continuer à coexister avec la législation communautaire, en particulier dans le domaine des pratiques anticoncurrentielles telles que les ententes et les abus de position dominante, en veillant à ce que les dispositions de ces législations nationales soient conformes au droit communautaire et que ce dernier prime en cas de conflit. Pour le second camp comprenant en particulier la Commission, l'UEMOA doit avoir une compétence exclusive pour légiférer dans le domaine des ententes, des abus de position dominante et des aides d'État, afin d'éviter les conflits de normes et de procédures, les législations nationales ne pouvant porter que sur les autres domaines du droit de la concurrence qui appartiennent à la compétence résiduelle des États, telle que la concurrence déloyale.

Saisie à titre consultatif par la Commission de l'UEMOA de la divergence de vues qui l'opposait aux experts des États membres de l'UEMOA, la Cour de Justice de l'UEMOA a tranché en faveur de la Commission dans son avis $n^{\circ} 003 / 2000$ du 27 juin 2000 concernant l'interprétation des articles du Traité UEMOA relatifs aux règles de concurrence dans l'Union ${ }^{64}$. En conséquence, estime la Cour, les États membres ne peuvent exercer de compétences partagées ou concurrentes dans ce domaine, comme le voudrait d'après ses analyses le principe de la double barrière appliquée en droit communautaire européen ${ }^{65}$ (par opposition au principe de la simple barrière choisie selon elle par le Traité de l'UEMOA), exception faite des prescriptions formelles des autorités communautaires associant ces Etats à l'exercice de cette compétence dévolue à l'Union ${ }^{66}$.

Cet avis de la Cour de justice de l'UEMOA a été pris en compte dans son principe par le législateur de l'UEMOA dans les règles de concurrence édictées le 23 mai 2002, plus particulièrement par la directive $n^{\circ}$ 02/2002/CM/UEMOA relative à la coopération entre la Commission et les structures nationales de concurrence des États membres dans la lutte contre les pratiques anticoncurrentielles ${ }^{67}$. Toutefois, dans son aménagement concret, un tel principe a été amené à connaître quelques assouplissements du fait du souci du législateur de l'UEMOA de concilier la compétence exclusive de la Commission et la nécessité de permettre une surveillance efficace des marchés par les structures nationales de concurrence ${ }^{68}$.Plus précisément, si l'édiction des règles de fond est réservée aux structures communautaires, on note dans la mise en œuvre des règles de fond un partage des fonctions d'enquête entre les structures nationales de concurrence et la Commission ${ }^{69}$, même si les fonctions d'instruction et de décision sont monopolisées par la Commission ${ }^{70}$.

Après avoir mis en exergue le débat autour de la consécration de l'exclusivité de la compétence de l'UEMOA en matière de la concurrence qui d'ailleurs est de nature jurisprudentielle, nous allons voir à présent l'exercice de cette compétence par l'Union.

\section{2 -La compétence exclusive de la commission consacrée par la jurisprudence UEMOA}

Organe politique ${ }^{71}$, la Commission est avant tout chargée de la mise en œuvre de la politique de concurrence telle que définie dans l'Union. Dans ce cadre, l'option prise en vue de la réalisation des objectifs économiques détermine l'étendue de ses compétences. Dès lors, avec la centralisation de l'essentiel des compétences matérielles au niveau de l'Union, il n'est pas surprenant que la Commission soit la pièce maîtresse de l'application du droit communautaire de la concurrence en UEMOA.

En Afrique centrale, on retrouve ce système centralisé d'application du droit communautaire avec un organe de contrôle régional et une Cour arbitrale ${ }^{72}$. La compétence incontestable de la Commission, qui lui est reconnue par le traité ${ }^{73}$, se manifeste à tous les stades de la procédure de mise en œuvre du droit communautaire.

L'analyse du règlement $n^{\circ} 3 / 2002 / U E M O A$ qui régit les procédures applicables aux ententes et abus de position dominante montre que la Commission joue un rôle central dans la conduite des enquêtes. Ce quasimonopole se manifeste sous différents aspects. 
Le droit de saisine appartient concurremment à la Commission qui peut s'autosaisir, aux particuliers victimes de pratiques anticoncurrentielles et aux États membres ${ }^{74}$. Ces derniers, dans le cadre de leur mission générale de «veille permanente sur le fonctionnement du marché » ${ }^{75}$, peuvent saisir directement la Commission ou lui transmettre les plaintes des personnes physiques habilitées à la saisir.

Une fois saisie, la Commission, seule ou avec l'appui des autorités internes, procède à des enquêtes avec demandes de renseignement adressées aux entreprises en cause ou à des vérifications dans les États membres pour établir la véracité des faits ${ }^{76}$. Elle doit, dans l'exercice de ses compétences, " veiller à respecter les droits fondamentaux des entreprises » et notamment le principe du contradictoire dans la phase de l'instruction ${ }^{77}$.Dans le cadre du partage des fonctions d'enquête entre les structures nationales de concurrence et la Commission, seule cette dernière peut connaître des pratiques que sont les aides d'Etat, les pratiques anticoncurrentielles concernant les monopoles et entreprises publiques et les pratiques anticoncurrentielles susceptibles d'avoir un effet sur les échanges entre Etats membres ${ }^{78}$.

En outre, cette compétence exclusive accordée à l'Union rend inapplicable toute législation de concurrence interne des Etats membres qu'elle soit antérieure ou qu'elle soit postérieure. Cependant, l'avis du 27 juin 2000 de la Cour de justice de l'UEMOA accorde une sorte de compétence résiduelle aux Etats membres notamment la répression pénale des actes anticoncurrentiels qui peuvent porter atteintes à la transparence du marché et à l'organisation de la concurrence qui constituera forcément une entorse au droit UEMOA. Il faut signaler également que «Le droit pénal de la concurrence des Etats qui ont la compétence retenue en cette manière devra en conséquence s'adapter au droit communautaire pour caractériser les infractions pénalement punissables $^{79} \gg$. Le droit national est éliminé par le droit communautaire, mais il subsiste dans la répression de l'infraction communautaire. ${ }^{80}$

Par ailleurs, cette exclusivité accordée à l'Union est décriée par une partie de la doctrine sur l'UEMOA. Cette doctrine soutient que cette exclusivité n'a pas de fondement valable dans le traité constitutif de l'UEMOA et n'est pas un principe d'ensemble retenu par ledit traité. C'est en vain qu'on recherche son fondement. L'article 5 du traité prévoit l'édiction par les organes de l'Union des prescriptions minimales et des réglementations-cadres qu'il appartient aux Etats membres de compléter en tant de besoin, sans précision des domaines concernés. ${ }^{81}$

Toutefois, la mise en œuvre de la législation communautaire de la concurrence incombe de manière principale à l'Union étant donné qu'elle bénéficie d'une compétence exclusive en la matière.

\section{4 -La mise en œuvre des normes de concurrence entre exclusivité et coopération}

Il ressort de l'avis $n^{\circ} 003 / 2000$ de la Cour de justice que l'application du droit communautaire aussi est centralisée. Deux instances communautaires sont principalement chargées de l'application du droit de l'UEMOA : la commission et la Cour de justice. Le rapprochement est net avec l'union européenne où ces deux institutions ont notablement contribué à préciser les contours du droit communautaire de la concurrence. Mais en dépit de cette centralisation au niveau de la commission et de la Cour de justice, il y a lieu d'évoquer d'autres normes communautaires dans l'espace UEMOA avec lesquelles l'Union devrait collaborer ainsi que certaines structures administratives ou judiciaires des Etats membres intervenant dans le domaine de la mise en œuvre desdites normes.

\section{1 - La complémentarité et la collaboration dans les attributions entre la Cour de justice et le juge national}

L'application du droit communautaire de la concurrence en matière juridictionnelle échappe à la thèse de la compétence exclusive des institutions communautaires pour l'application dudit droit. La Cour de justice UEMOA dispose certes d'importantes attributions, mais les juridictions nationales sont aussi amenées à connaître du droit communautaire de la concurrence.

La Cour de façon spécifique, a la compétence de connaitre de recours contre les décisions prises par la commission dans l'application du droit de la concurrence. L'article 90 du traité précise d'ailleurs nettement que la commission applique le droit communautaire de la concurrence sous le contrôle de la Cour de justice. Ainsi, une entreprise ou un Etat peut faire annuler une décision de la commission exemptant ou au contraire prohibant une entente, prononçant une amende ou déclarant illégale une aide publique ou, à l'inverse, la validant. Le recours en annulation peut porter sur le droit dérivé de la concurrence tel qu'un règlement ou une directive jugée contraire au traité. Il peut se doubler d'une action en responsabilité contre la commission ou l'Union pour le préjudice qui aurait pu résulter de l'acte incriminé. L'illégalité de l'acte est susceptible d'être soulevée par voie d'exception.Le rôle de la Cour prend ainsi un relief particulier en droit de la concurrence, en raison de l'institution d'un recours de pleine juridiction devant la Cour contre les décisions et sanctions prononcées en droit de la concurrence par la commission contre les entreprises. ${ }^{82}$

En dehors de ces hypothèses, l'application du droit communautaire relève de la compétence des juridictions nationales et les Etats membres utilisent à cet effet le même système institutionnel que pour 
l'application du droit interne. La solution est identique à celle du droit de l'union européenne : la compétence de la Cour de justice est une compétence d'attribution, se limitant aux prescriptions du traité et du droit dérivé, les juges nationaux agissant comme juges communautaires de droit commun. C'est l'expression même de l'application directe du droit communautaire, principe qui permet aux justiciables d'invoquer la norme communautaire devant le juge national. Une entreprise peut ainsi faire valoir directement contre une autre, devant les juridictions nationales, les règles de droit communautaire de la concurrence. ${ }^{83}$

La faible application du droit communautaire de la concurrence par les juridictions nationales dans l'espace UEMOA jusqu'à présent ne permet pas d'élaborer un bilan conséquent et d'entrevoir des pistes concrètes de réforme. Néanmoins, des risques réels apparaissent d'ores et déjà clairement quant aux divergences dans l'application uniforme du droit de la concurrence par les juridictions nationales et l'on pourra s'inspirer de certaines dispositions en droit de l'union européenne. Il est impératif de rendre le recours préjudiciel obligatoire pour l'appréciation de la légalité du droit dérivé par les juridictions nationales des Etats membres de l'UEMOA, comme dans l'union européenne.

La coopération entre les juridictions nationales et la commission de l'UEMOA peut constituer une autre voie d'unification de la jurisprudence. Les juridictions nationales, appliquant le droit communautaire de la concurrence, possèdent le pouvoir de faire des investigations dans le cadre de la résolution des litiges qui leur sont soumis. Pour aller plus loin, d'autres mesures peuvent être envisagées, telles que la demande d'avis à la commission, la possibilité pour celle-ci de présenter des observations écrites ou orales devant les juridictions nationales, quand elles statuent en droit communautaire de la concurrence. ${ }^{84}$

Les juridictions nationales européennes ne peuvent adopter des solutions qui vont à l'encontre d'une décision préexistante de la commission de l'union européenne ${ }^{85}$ ou d'une décision envisagée par elle dans une procédure.

Si nous avons vu ci-dessus, la relation de collaboration et de coopération qu'entretient la Cour de justice UEMOA avec les structures judiciaires nationales, qu'en est-il de la coexistence possible entre le droit UEMOA de la concurrence et d'autres normes d'organisations régionales dans l'espace de l'Union?

\section{2 - La coexistence des droits sous régionaux et internationaux de la concurrence avec le droit UEMOA}

Dans la sous-région, il faut noter qu'à côté de l'UEMOA, la CEDEAO aussi s'est dotée d'un droit de la concurrence et a élaboré plusieurs textes ${ }^{86}$ et la présence de l'Organisation pour l'harmonisation en Afrique du droit des affaires (OHADA) invite à tenir compte d'éventuels futurs actes uniformes règlementant le droit de la concurrence. En effet, L'OHADA envisage d'autres harmonisations juridiques, en particulier dans le domaine du droit du travail, du transport, des contrats etc... Mais surtout, l'OHADA envisage d'autres harmonisations juridiques, en particulier dans le domaine du droit de la concurrence, comme l'a laissé entendre son Conseil des ministres qui s'est tenu les 22 et 23 mars 2001 à Bangui ${ }^{87}$. Ainsi, il y a des risques de recoupement des droits de la concurrence de l'UEMOA et de l'OHADA et en particulier des règles de fond émanant de ces deux organisations $^{88}$.

En outre, il y a des risques de recoupement des droits de la concurrence de l'UEMOA de la CEDEAO, de l'OHADA, et dans une moindre mesure, ceux des Etats membres, puisque les droits de la concurrence des Etats ne doivent pas subsister en principe dans les domaines couverts par le droit UEMOA. Malgré l'existence de dispositions dans les Traités favorisant la coopération et la concertation entre les différentes organisations par rapport aux risques d'incompatibilité entre les droits de la concurrence de ces organisations, l'émergence effective d'un droit de la concurrence au niveau de la CEDEAO pourrait poser de réels conflits de compétence.

Toutefois, il apparaît que le mieux par rapport à ces problèmes de compatibilité entre les droits de la concurrence de l'UEMOA et de l'OHADA est d'avoir à les prévenir plutôt qu'à les guérir. De ce point de vue, on peut envisager, comme solution de prévention de ces problèmes, le recours à la pratique systématique de la concertation et de la collaboration au cours des procédures d'élaboration et d'application des règles de l'UEMOA et de l'OHADA ${ }^{89}$.

Dans le cas d'espèce, il serait plus réaliste que le législateur de l'OHADA, à défaut de renoncer à son idée d'édicter un droit de la concurrence venant s'ajouter à celui des organisations « transnationales » existantes (en particulier l'UEMOA), s'inspire profondément des règles déjà établies au niveau de l'UEMOA. Dans cette optique, l'OHADA pourra se borner à énoncer quelques principes et règles générales en matière de concurrence, en laissant aux organisations régionales inférieures regroupant ses États membres (en particulier l'UEMOA et la Communauté économique et monétaire d'Afrique centrale CEMAC -) l'adoption d'une législation plus détaillée dans ce domaine. Les États membres de l'UEMOA pourront peser de tout leur poids au sein de l'OHADA pour obtenir une telle solution. ${ }^{90}$

\section{CONCLUSION}

En guise de conclusion, il faut dire que dans le Traité de l'UEMOA, tout comme dans celui de l'Union européenne, le principe de la libre concurrence est un des principes cardinaux qui sous-tendent l'instauration 
d'un marché commun basé sur la libre circulation des personnes, des biens, des services et le droit d'établissement. La maîtrise de ces principes par les acteurs au développement sera d'un grand apport à la consolidation du tissu économique des Etats membres.

Il faut également instaurer une culture de la concurrence dans les Etats membres, qui est un déterminant essentiel dans la définition des modalités de coopération entre les nationaux et le niveau communautaire. Si une solide culture de la concurrence existe dans les États membres avec des institutions fortes, capables de mettre en œuvre une politique de la concurrence, il est concevable de reconnaitre des pouvoirs étendus aux autorités nationales dans la mise en œuvre du droit communautaire de la concurrence.

Aussi, il faudrait rappeler que la mise en œuvre de droit de la concurrence communautaire requiert une volonté politique manifeste et a besoin d'un soutien solide pour éclore, prospérer et devenir une réalité.

Il faudrait enfin, un bon aménagement des institutions de la concurrence pour tenir compte de la distinction entre les pratiques relevant de la commission de l'UEMOA et les pratiques relevant de la compétence des autorités nationales.

\section{REFERENCESBIBLIOGRAPHIQUES}

[1] INS/C. UEMOA : RSM juin 2016. Extraits Traité de l'UEMOA.Rapport intégral sur l'examen de la politique de la concurrence de l'UEMOA, du Bénin et du Sénégal (CNUCED juillet 2007) Note 3 et 4 de l'annexe $\mathrm{n}^{\circ} 1 \mathrm{du}$ règlement $\mathrm{n}^{\circ} 03 / 2002 / \mathrm{CM} / \mathrm{UEMOA}$.

[2] Règlement $n^{\circ}$ 02/2002/CM/UEMOA » pour « règlement $n^{\circ}$ 02/2002/CM/UEMOA relatif aux pratiques anticoncurrentielles à l'intérieur de l'Union économique et monétaire ouest africaine »;

[3] Règlement $n^{\circ}$ 03/2002/CM/UEMOA » pour « règlement $n^{\circ}$ 03/2002/CM/UEMOA relatif aux procédures applicables aux ententes et abus de position dominante à l'intérieur de l'Union économique et monétaire ouest africaine »;

[4] Règlement $n^{\circ}$ 04/2002/CM/UEMOA » pour « règlement $n^{\circ}$ 04/2002/CM/UEMOA relatif aux aides d'Etat à l'intérieur de l'Union économique et monétaire ouest africaine et aux modalités d'application de l'article 88 (c) du Traité $»$;

[5] Directive $n^{\circ}$ 01/2002/CM/UEMOA » pour «directive $n^{\circ}$ 01/2002/CM/UEMOA relative à la transparence des relations financières d'une part entre les Etats membres et les entreprises publiques, et d'autre part entre les Etats membres et les organisations internationales ou étrangères »;

[6] Directive $n^{\circ}$ 02/2002/CM/UEMOA » pour « directive $n^{\circ}$ 02/2002/CM/UEMOA relative à la coopération entre la Commission et les structures nationales de concurrence des Etats membres pour l'application des articles 88, 89 et 90 du Traité de l'UEMOA ».

[7] Traité de Rome version consolidée

[8] Décision $\mathrm{n}^{\circ}$ 0157/2007/PCOM/UEMOA portant création et organisation des services de la Commission de l'UEMOA, inédit.

[9] Décision $\mathrm{n}^{\circ} 345 / 2008 / \mathrm{PCOM} / \mathrm{UEMOA}$ portant organisation du département du marché régional, du commerce, de la concurrence et de la coopération, inédit.

[10] UEMOA, Cour de justice, textes fondamentaux et jurisprudence, édition giraf, 2003, pp. 156 et suivant.

[11] « demande d'avis de la Commission de l'UEMOA relative à l'interprétation des articles 88, 89 et 90 du Traité relatifs aux règles de concurrence dans l'Union », in Cour de justice de l'UEMOA, « Recueil de la jurisprudence de la Cour », Cour de justice, Ouagadougou, février 2002,

[12] L'Acte additionnel $\mathrm{n}{ }^{\circ} \mathrm{A} / \mathrm{SA} 1 / 06 / 08$ portant adoption des Règles Communautaires de la Concurrence et de leurs modalités d'application au sein de la CEDEAO ;

[13] L'Acte Additionnel n ${ }^{\circ} \mathrm{A} / \mathrm{SA} / \mathrm{SA} .2 / 06 / 08$ Portant création et attributions et fonctionnement de l'Autorité Régionale de la Concurrence (ARC) de la CEDEAO. Le Cadre Régional de Politique de la Concurrence.

\section{REVUES}

[14] MÉLÉDO-BRIAND (D.), « Droit de la concurrence, droit constitutionnel substantiel de l'Union européenne », RTD com., avril-juin 2004,

[15] Henri CHAVRIER et al., « Chronique générale de jurisprudence communautaire : janvier 1997-décembre 1998.

[16] Troisième partie : Les règles de concurrence applicables aux entreprises », Revue du Marché Commun et de l’Union européenne, $n^{\circ} 436$, mars 2000, p. 179-196;

[17] Jean-Michel COUMES et Jérôme BROCHE, « La proposition de réforme du droit communautaire de la concurrence : le principe et ses conséquences », Revue du Marché Commun et de l'Union européenne, $\mathrm{n}^{\circ}$ 451, septembre 2001,

[18] Christian GARBAR, « Aides d'Etat : pratique décisionnelle de la Commission de la Communauté européenne (1990-1994) », 2e partie, in Revue du Marché Commun et de l'Union Européenne, $n^{\circ} 384$, janvier 1995, 
[19] Françoise HAUTFENNE, «Approche comparative des concepts d'entente et d'abus de position dominante dans le cadre de l'U.E. et de l'U.E.M.O.A. », Revue burkinabè de droit, n ${ }^{\circ} 34,1998$

[20] Etienne CEREXHE, «Le rôle de la Cour de justice des Communautés européennes dans le domaine de la concurrence », in Centre d'études européennes et de l'intégration (CEEI), "Actes du colloque de Ouagadougou des 16 et 17 décembre 1999 portant sur le thème «La libéralisation de l'économie dans le cadre de l'intégration régionale : le cas de l'UEMOA », Imprimerie Presses Africaines, Ouagadougou, 2001 ,

[21] M. Bakhoum, «L'articulation du droit communautaire et des droits nationaux de la concurrence dans l’Union Économique et Monétaire Ouest-Africaine(UEMOA) », op. cit. (Note 12),

[22] Mor Bakhoum, «Cohérence institutionnelle et effectivité d'une politique régionale de la concurrence : le cas de l'union économique et monétaire ouest-africaine (UEMOA) », De Boeck Supérieur|Revue internationale de droit économique, 2011,

[23] V. J. - P. Viennois, « la portée du droit communautaire de la concurrence et le mythe du champ d'application exclusive du droit national », RTD, Com. 2002

[24] A. PAPPARLADO, « Les relations entre le droit communautaire et les droits internes nationaux », RID éco, 1995,

[25] G. Isaac, « la primauté du droit communautaire », Rev juris. Com. № spécial, nov 1987, P. 16.

[26] W MOESCHEL, «Harmonisation ou concurrence entre les différents droits de la concurrence », RTD com 2006,

[27] KENFACK (Y.), «Réglementation communautaire de la concurrence et processus d'intégration économique en Afrique centrale », Genève, CNUCED, UNCTAD/ITCD/CLP/Misc.15, 2000,

[28] DIENG (P.-A.), " Les procédures contentieuses dans la législation communautaire de l'UEMOA », Actes du séminaire sous régional de sensibilisation sur le droit communautaire UEMOA, Ouagadougou, 6 octobre 2003,

[29] Ayawa Améhia TSKADI, «De la compétence exclusive de l’Union en droit de la concurrence dans l'espace UEMOA », Revue droit uniforme africain, Actualité trimestrielle de droit et de jurisprudence $\mathrm{N}^{\circ}$ 005, 2011

[30] P. Meunier, «La Cour de justice des communautés européennes et l'applicabilité des règles de concurrence du traité CECA », RTD eur, 1996, P. 243 ; CJCE, 6 avril 1962, aff, 13/61, Rec.,

[31] Joël BOUDANT, "L'activité anti-dumping communautaire (janvier-octobre 1994) », Revue du Marché Commun et de l'Union Européenne, n 387, avril 1995,

[32] DEMBA Boubacar BA, " Le problème de la compatibilité entre l'UEMOA et l'OHADA », in Centre d'études européennes et de l'intégration (CEEI), « Actes du colloque de Ouagadougou des 16 et 17 décembre 1999 portant sur le thème «La libéralisation de l'économie dans le cadre de l'intégration régionale : le cas de l'UEMOA».

[33] COULIBALY abousaïb, « le droit de la concurrence de l'union économique et monétaire ouest africaine revue burkinabé de droit, $\mathrm{n}^{\circ}$ 43-44, 1er et 2ème semestres, Ouagadougou 2003.

\section{OUVRAGES}

[34] Ahmed SILEM et Jean-Marie ALBERTINI (dir.), « Lexique. Economie », 5e éd, Dalloz, Paris, 1995.

[35] Paul ROBERT et alii, «Le Petit Robert (Dictionnaire alphabétique et analogique de la langue française) Dictionnaires Robert, 30e éd, Paris, 1976.

[36] Jean-Marc MOUSSERON et Véronique SELINSKY, « Le droit français nouveau de la concurrence »,2e éd, Litec, Paris, 1988.

[37] Gérard CAS, Roger BOUT, Etienne PETIT, « Lamy droit économique (1996) : concurrence, distribution, consommation », Lamy, Paris, 1995.

[38] Jean-Claude GAUTRON, « Droit européen », op. cit.,

[39] Jean-Paul KEPPENNE, « Guide des aides d'Etat en droit communautaire (Réglementation, jurisprudence et pratique de la Commission) », Bruylant, Bruxelles, 1999.

[40] Etienne CEREXHE et Louis le HARDY de BEAULIEU, « Introduction à l'union économique ouest africaine », Bruxelles, De Boeck et Larcier, 1997.

[41] Philippe TIGER, «Le droit des affaires en Afrique (OHADA) », Presses universitaires de France, 1 janv. 1999.

ACTES COLLOQUES

[42] Jean-Paul KEPPENNE, « Le rôle respectif des autorités (administratives et juridictionnelles) communautaires et nationales dans la mise en œuvre d'un droit communautaire de la concurrence : l'expérience européenne et les projets de l'UEMOA », communication au colloque organisé par le Centre d'études européennes et de l'intégration (CEEI) de l'UFR/Sciences juridiques et politiques (UFR/SJP) de 
l'Université de Ouagadougou sur « Le droit de la concurrence et de la consommation dans l'espace UEMOA », Ouagadougou, 5-8 février 2002 (publication prévue dans le cadre des actes dudit colloque).

\section{[43] SITES INTERNETS}

[44] www.izf.net

[45] www.uemoa.int

[46] http:// r0.unctad.org/en/subsites/cpolicy/docs/afriquecent.pdf.

[47] http://droit.francophonie.org/doc/html/znao/doc/dcum/ 2003dfznaodcum12.html.

1 - Source: INS/C. UEMOA : RSM juin 2016.

2 - Nous empruntons cette expression au Professeur François RIGAUX, qui l'utilise dans son ouvrage «Introduction à la science du droit », éd. Vie Ouvrière, Bruxelles et Paris, 1974, (chapitre 28 " La juridisation des relations sociales »), p. 101 et $\mathrm{s}$. La juridisation est la pénétration des règles de droit dans des relations sociales de plus en plus nombreuses, relations qui sont ainsi de mieux en mieux enserrées par le droit.

3 - Article 89 du Traité de l'UEMOA.

4 - Voy Rapport intégral sur l'examen de la politique de la concurrence de l’UEMOA, du Bénin et du Sénégal (CNUCED juillet 2007), P. 8.

5 - Pour plus de précisions, voir notamment Ahmed SILEM et Jean-Marie ALBERTINI (dir.), «Lexique. Economie ", 5e éd, Dalloz, Paris, 1995, p. 148-150.

6 - Pour plus de précisions, Ahmed SILEM et Jean-Marie ALBERTINI (sous la direction de), "Lexique. Economie", op. cit., p. 148-150.

7 - Yves SERRA, «Le droit français de la concurrence », Dalloz, Paris, 1993, p. 9.

8 - Pour cette définition, voir notamment : - Paul ROBERT et alii, « Le Petit Robert (Dictionnaire alphabétique et analogique de la langue française) ", Dictionnaires Robert, Paris, 1976, 30e éd., p. 322 ; - Jean-Marc MOUSSERON et Véronique SELINSKY, «Le droit français nouveau de la concurrence », Litec, Paris, 1988, 2e éd., p. 7-8 ; - Yves SERRA, «Le droit français de la concurrence », op cit, p. 1-2.

9 - Rapport intégral sur l'examen de la politique de la concurrence de l'UEMOA, du Bénin et du Sénégal (CNUCED juillet 2007) op cit, P. 8.

10 -Sur le droit communautaire européen des ententes, principalement régies par l'article 81 (ex-article 85) du Traité CE, voir notamment : - Gérard CAS, Roger BOUT, Etienne PETIT, « Lamy droit économique (1996) : concurrence, distribution, consommation », Lamy, Paris, 1995, p. 595-629 ; - Henri CHAVRIER et al., "Chronique générale de jurisprudence communautaire : janvier 1997-décembre 1998.

Troisième partie : Les règles de concurrence applicables aux entreprises », Revue du Marché Commun et de l'Union européenne, $\mathrm{n}^{\circ}$ 436, mars 2000, p. 179-196 ; - Jean-Michel COUMES et Jérôme BROCHE, « La proposition de réforme du droit communautaire de la concurrence : le principe et ses conséquences ", Revue du Marché Commun et de l'Union européenne, $\mathrm{n}^{\circ} 451$, septembre 2001, p. 546-552 ;

11 - Note 4 de l'annexe $n^{\circ} 1$ du règlement $n^{\circ} 03 / 2002 / C M / U E M O A$.

12 - Une pareille interdiction des abus de position dominante est également faite en droit européen de la concurrence et dans des droits nationaux de la concurrence de pays membres de l'espace UEMOA.

Sur le droit communautaire européen des abus de position dominante, principalement régis par l'article 82 (ex-article 86) du Traité de Rome, voir notamment : - Gérard CAS, Roger BOUT, Etienne PETIT, « Lamy droit économique (1996) : concurrence, distribution, consommation », op. cit, p. 630-646 ; - Henri CHAVRIER et al., "Chronique générale de jurisprudence communautaire : janvier 1997- décembre 1998.

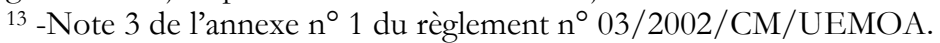

14 - Une semblable interdiction des aides publiques existe également au niveau des règles de concurrence de l'Union européenne. Sur le droit communautaire européen d'interdiction des aides publiques, principalement formulée par les articles 87 à 89 (ex-articles 92 à 94) du Traité CE, voir notamment : - Gérard CAS, Roger BOUT, Etienne PETIT, «Lamy droit économique (1996) : concurrence, distribution, consommation », op. cit., p. 679-693 ; - JeanClaude GAUTRON, «Droit européen », op. cit., p. 181-182 ; - Christian GARBAR, « Aides d'Etat : pratique décisionnelle de la Commission de la Communauté européenne (1990-1994) », 2e partie, in Revue du Marché Commun et de l'Union Européenne, n 384, janvier 1995, p. 36-45 ; - Jean-Paul KEPPENNE, « Guide des aides d'Etat en droit communautaire (Réglementation, jurisprudence et pratique de la Commission) », Bruylant, Bruxelles, 1999, p. 693. 
15 - Ces trois règlements et ces deux directives qui n'avaient pas encore été publiés au Bulletin Officiel de l'UEMOA au moment de la finition du présent article se trouvent sur le réseau Internet, au site « http : // www.uemoa.int », en cliquant sur l'item « actes» de la page d'accueil.

16 - CUTS Centre for Compétition, Investment \& Economic Régulation (CUTS CCIER) CUTS International, «Vers des régimes efficients de la concurrence par le renforcement des capacités dans des pays sélectionnés d'Afrique de l'Ouest (Projet 7Up4) », Document préliminaire Sénégal sur les scénarios de concurrence, Janvier 2009, P 22.

17 - Voir notamment Etienne CEREXHE et Louis le HARDY de BEAULIEU, " Introduction à l'union économique ouest africaine », op. cit, p. 77.

18 - Article 83 (ex-article 87) du Traité de Rome version consolidée.

19 - Voir notamment Françoise HAUTFENNE, "Approche comparative des concepts d'entente et d'abus de position dominante dans le cadre de l'U.E. et de l'U.E.M.O.A. », Revue burkinabè de droit, n 34, 1998, pp. 173199.

20 - Article 90 du Traité de l'UEMOA. Voir notamment en ce sens : - Etienne CEREXHE et Louis le HARDY de BEAULIEU, «Introduction à l'union économique ouest africaine », op. cit., p. 77 ; - Jean-Paul KEPPENNE, «Le rôle respectif des autorités (administratives et juridictionnelles) communautaires et nationales dans la mise en œuvre d'un droit communautaire de la concurrence : l'expérience européenne et les projets de l'UEMOA », communication au colloque organisé par le Centre d'études européennes et de l'intégration (CEEI) de l'UFR/Sciences juridiques et politiques (UFR/SJP) de l'Université de Ouagadougou sur « Le droit de la concurrence et de la consommation dans l'espace UEMOA », Ouagadougou, 5-8 février 2002 (publication prévue dans le cadre des actes dudit colloque), p. 12.

21 - Voir en particulier les articles 8 à 11 du règlement no 03/2002/CM/UEMOA pour les ententes et les abus de position dominante, l'article $5 \mathrm{du}$ règlement $\mathrm{n}^{\circ}$ 04/2002/CM/UEMOA pour les aides d'Etat, l'article 6 paragraphe 6.2 alinéa 3 du règlement $n^{\circ}$ 02/2002/CM/UEMOA pour les monopoles et entreprises publiques, l'article 2 de la directive $n^{\circ}$ 01/2002/CM/UEMOA pour la transparence des relations entre les Etats et les entreprises publiques.

22 - Voir en particulier les articles 12 à 14 du règlement $n^{\circ}$ 03/2002/CM/UEMOA pour les ententes et les abus de position dominante, l'article 22 paragraphe 22.2 du règlement $n^{\circ}$ 04/2002/CM/UEMOA pour les aides d'Etat.

23 - Voir notamment l'article 3 paragraphe 3.1 et l'article 4 paragraphe 4.1 du règlement $n^{\circ}$ 03/2002/CM/UEMOA pour les ententes et les abus de position dominante.

24 - Article 17 du règlement $\mathrm{n}^{\circ}$ 03/2002/CM/UEMOA pour les ententes et les abus de position dominante, article 23 du règlement $\mathrm{n}^{\circ} 04 / 2002 / \mathrm{CM} / \mathrm{UEMOA}$ pour les aides d'Etat.

25 - Articles 18 à 21 du règlement $n^{\circ}$ 03/2002/CM/UEMOA pour les ententes et les abus de position dominante, articles 13 et 23 du règlement $n^{\circ}$ 04/2002/CM/UEMOA pour les aides d'Etat.

26 -Voir notamment l'article 3 paragraphe 3.1 du règlement $n^{\circ}$ 03/2002/CM/UEMOA pour les ententes et les abus de position dominante, l'article 7 paragraphes 7.2, 7.3 et 7.6, l'article 10 paragraphes 10.2 et 10.3 du règlement $n^{\circ}$ 04/2002/CM/UEMOA pour les aides d'Etat.

27 - Articles 6 et 7 du règlement $n^{\circ}$ 03/2002/CM/UEMOA pour les ententes et les abus de position dominante,

28 - Voir notamment l'article 5 du règlement $n^{\circ}$ 03/2002/CM/UEMOA pour les ententes et les abus de position dominante, les articles 14 et 16 du règlement $n^{\circ}$ 04/2002/CM/UEMOA pour les aides d'Etat.

29 - Article 22 et 23 du règlement $n^{\circ} 03 / 2002 / C M / U E M O A$ pour les ententes et les abus de position dominante.

30 - Voir les articles 24 et 89 du Traité de l'UEMOA et les articles 3 et 6 du règlement n 03/2002/CM/UEMOA.

31 - CUTS Centre for Compétition, Investment \& Economic Régulation (CUTS CCIER) CUTS International, «Vers des régimes efficients de la concurrence par le renforcement des capacités dans des pays sélectionnés d'Afrique de l'Ouest (Projet 7Up4) », op cit, p.23.

32 - FOFANA Ramata (Juge à la Cour de Justice de l'UEMOA), «Le droit de la concurrence de l'UEMOA », article dans le manuel de formation des magistrats en droit public des affaires UEMOA/CEMAC du 20 au 31 octobre 2008, P.88.

33 -Article 90 du Traité de l'UEMOA.

34 - Article 31 du règlement nº 03/2002/CM/UEMOA.

35 - Idem. Sur le rôle du juge communautaire dans l'application des règles de concurrence, voir notamment Etienne CEREXHE, «Le rôle de la Cour de justice des Communautés européennes dans le domaine de la concurrence », in Centre d'études européennes et de l'intégration (CEEI), "Actes du colloque de Ouagadougou des 16 et 17 décembre 1999 portant sur le thème «La libéralisation de l'économie dans le cadre de l'intégration régionale : le cas de l'UEMOA », Imprimerie Presses Africaines, Ouagadougou, 2001, p. 85-96.

36 - Article 6 paragraphe 6.4. du règlement $n^{\circ}$ 02/2002/CM/UEMOA.

37 - Article 28 paragraphe 28.4 du règlement $n^{\circ} 03 / 2002 / \mathrm{CM} / \mathrm{UEMOA}$.

38 - Article 29 du règlement $n^{\circ} 04 / 2002 / \mathrm{CM} / \mathrm{UEMOA}$.

39 - Pour les précisions sur ces demandes et notifications, voir les articles 8 et 9 du règlement $n^{\circ}$ 03/2002/CM/UEMOA.

40 - Article 15 paragraphes 15.1; 15.2 et 15.3 du règlement nº3/2002/CM/UEMOA. 
41 - Pour de plus amples informations sur les conditions d'introduction de ces plaintes, voir les articles 12 et 13 du règlement $\mathrm{n}^{\circ} 03 / 2002 / \mathrm{CM} / \mathrm{UEMOA}$.

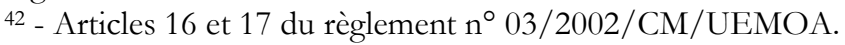

43 - Dans ce domaine également, les solutions retenues ressemblent aux solutions du droit européen. Pour plus d'informations allant dans ce sens, voir notamment : Jean-Paul KEPPENNE, « Le rôle respectif des autorités (administratives et juridictionnelles) communautaires et nationales dans la mise en œuvre d'un droit communautaire de la concurrence : l'expérience européenne et les projets de l'UEMOA », op. cit., p. 27-30

44 - Articles 16 et 18 du règlement nº 04/2002/CM/UEMOA.

45 - Article 20 du règlement $n^{\circ}$ 04/2002/CM/UEMOA.

46 - Article 24, paragraphe 24.1, du règlement n 04/2002/CM/UEMOA. Il s'agit des mesures suivantes : la publication, sur recommandation au Conseil, d'un communiqué, éventuellement assorti d'informations supplémentaires sur la situation de l'État concerné ; le retrait, annoncé publiquement, des mesures positives dont bénéficie éventuellement l'État membre ; la recommandation à la Banque ouest africaine de développement (BOAD) de revoir sa politique d'intervention en faveur de l'État membre concerné ; la suspension partielle ou totale des aides financières et concours de l'Union à l'État membre concerné. De même, la Commission peut infliger à l'entreprise qui continue de bénéficier de l'aide en dépit d'une décision de la Commission une amende allant jusqu'au double du montant de l'aide octroyée (Article 24, paragraphe24.2, du règlement nº 04/2002/CM/UEMOA).

47 - Ces sanctions sont prévues dans le Règlement n $03 /$ 2002/CM/UEMOA. L'article 22 de ce règlement fixe les montants de ces amendes à l'égard des entreprises et associations d'entreprises de 500000 FCFA.

48 - Voir M. Bakhoum, "L'articulation du droit communautaire et des droits nationaux de la concurrence dans l'Union Économique et Monétaire Ouest-Africaine(UEMOA)», op. cit. (Note 12), pp. 228-243.

49 - Voir Décision n 0157/2007/PCOM/UEMOA portant création et organisation des services de la Commission de l'UEMOA, inédit.

50 - Voir Décision n 345/2008/PCOM/UEMOA portant organisation du département du marché régional, du commerce, de la concurrence et de la coopération, inédit.

51 - Il faut rappeler également que la commission qui est compétente à connaitre les pratiques anticoncurrentielles des Etats membres avec laquelle collabore la direction de la concurrence ne dispose également que de 3 agents.

52 - Mor Bakhoum, «Cohérence institutionnelle et effectivité d'une politique régionale de la concurrence : le cas de l'union économique et monétaire ouest-africaine (UEMOA)», De Boeck Supérieur | Revue internationale de droit économique, 2011, PP. 8-9.

53 - Ibid, P. 10.

54 - Voir M. Bakhoum, «L'articulation du droit communautaire et des droits nationaux de la concurrence dans l'Union Économique et Monétaire Ouest-Africaine (UEMOA)», op. cit. (Note 12), p. 229 et suiv.

55 - C'est le cas de la collaboration de la Commission nationale de la concurrence du Sénégal avec la Commission de l'UEMOA. C'est plutôt avec la Direction du Commerce Intérieur (DCI) que la Commission travaille lors de ses enquêtes sur le terrain.

56- Mor Bakhoum, «Cohérence institutionnelle et effectivité d'une politique régionale de la concurrence : le cas de l'union économique et monétaire ouest-africaine (UEMOA)», op cit, P.13.

57 - Pour dire Ouagadougou, capitale du Burkina Faso où la Commission a son siège.

58 - UEMOA, Cour de justice, textes fondamentaux et jurisprudence, édition giraf, 2003, pp. 156 et suivant.

59 - La question du champ d'application du droit communautaire de la concurrence a été également soulevée en droit CE, V. J. - P. Viennois, «la portée du droit communautaire de la concurrence et le mythe du champ d'application exclusive du droit national», RTD, Com. 2002, P.1; A. PAPPARLADO, " Les relations entre le droit communautaire et les droits internes nationaux », RID éco, 1995, pp 123 et suivant.

${ }^{60}$ - Sur ce principe voir G. Isaac, « la primauté du droit communautaire », Rev juris. Com. N spécial, nov 1987, P. 16.

61 - Gaz. Pal. , 1964, 2, 191. Voir aussi arrêt simmenthal du 9 mars 1978, aff, 106/77, Rec, P. 629 : « Issu d'une source autonome, le droit né du traité ne pourrait, en raison de sa nature spécifique originale, se voir judiciairement opposer un texte interne quel qu'il soit, sans perdre son caractère communautaire et sans que soit mise en cause la base juridique de la communauté elle-même, le transfert opéré par les Etats, de leur ordre juridique interne au profit de l'ordre juridique communautaire, des droits et obligations correspondant aux dispositions du traité, entraine donc une limitation définitive de leurs droits souverains contre laquelle ne saurait prévaloir un acte unilatéral ultérieur incompatible avec la notion de communauté».

62 - W MOESCHEL, "Harmonisation ou concurrence entre les différents droits de la concurrence », RTD com 2006, pp. 37 et s, fait le constat de l'asymétrie entre les réalités économiques qui se globalisent et le droit destiné à les réguler, qui demeure national ou, dans quelques cas, régional. Il envisage plusieurs solutions possibles pour la surmonter. 
63 - Voir notamment la « demande d'avis de la Commission de l’UEMOA relative à l'interprétation des articles 88, 89 et 90 du Traité relatifs aux règles de concurrence dans l'Union », in Cour de justice de l'UEMOA, « Recueil de la jurisprudence de la Cour », Cour de justice, Ouagadougou, février 2002, p. 121.

64 - « Avis nº 003/2000 du 27 juin 2000 relatif à l'interprétation des articles 88, 89, 90 du Traité sur les règles de concurrence de l'Union », in Cour de justice de l'UEMOA, « Recueil de la jurisprudence de la Cour », op. cit., p. 119132.

65 - A ce propos, comme le note Jean-Claude GAUTRON, « la notion de concurrence, au sens communautaire, est limitée aux actions des Etats et comportements des entreprises susceptibles d'affecter le commerce entre les Etats membres ». Aussi, « le droit communautaire de la concurrence laisse subsister des droits nationaux de la concurrence qui s'appliquent aux actions et comportements n'ayant pas d'effet sur le commerce intra-communautaire, c'est-à-dire limités aux seuls marchés nationaux. Dans les autres cas, les législations nationales sont subordonnées aux règles communautaires (C.J.C.E., 15 févr. 1969, Walt Wilhem). En cas de conflit, le droit communautaire prévaut (C.J.C.E., 13 févr. 1969, Bayer A.G.) » (Jean-Claude GAUTRON, «Droit européen », op. cit., p. 176).

66 - Selon la Cour de justice de l'UEMOA, « les Etats membres restent somme toute compétents en toute exclusivité, pour prendre toutes dispositions pénales réprimant les pratiques anticoncurrentielles, les infractions aux règles de transparence du marché et même à l'organisation de la concurrence ».

67 - Voir en particulier les références faites à cet avis dans les visas de la directive n 02/2002/CM/UEMOA.

68 - Voir en particulier les motivations avancées par le législateur dans les visas de la directive $\mathrm{n}^{\circ}$ 02/2002/CM/UEMOA.

69 - Voir en particulier l'article 3 et l'article 5 paragraphes 5.1, 5.2 et 5.3 de la directive n ${ }^{\circ}$ 02/2002/CM/UEMOA.

70 - Article 5 paragraphe 5.4 de la directive $\mathrm{n}^{\circ}$ 02/2002/CM/UEMOA.

71 - Il faut préciser que les membres de la Commission sont désignés par la Conférence des chefs d’État et de gouvernement comme le prévoit l'article 27 du traité UEMOA. Pour ce qui est de la composition, des compétences, de la nomination et de la révocation des membres de la Commission, voir les articles 26 à 34 du traité UEMOA.

72. Le droit de la concurrence de la CEMAC sera mis en œuvre par un organe de contrôle régional et une Cour arbitrale. L'organe de contrôle régional est constitué de l'Organe de surveillance de la concurrence (OSC) qui regroupe en son sein le Secrétariat général de l'UDEAC et le Conseil régional de la concurrence (CRC). La Cour arbitrale a pour mission d'examiner les recours exercés contre les décisions du Conseil régional de la concurrence. Voir à ce sujet KENFACK (Y.), "Réglementation communautaire de la concurrence et processus d'intégration économique en Afrique centrale ", Genève, CNUCED, UNCTAD/ITCD/CLP/Misc.15, 2000, p. 20, disponible sur : http:// r0.unctad.org/en/subsites/cpolicy/docs/afriquecent.pdf.

73 - Article 90 du traité UEMOA.

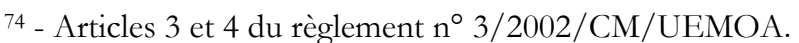

75 - DIENG (P.-A.), «Les procédures contentieuses dans la législation communautaire de l'UEMOA », Actes du séminaire sous régional de sensibilisation sur le droit communautaire UEMOA, Ouagadougou, 6 octobre 2003, disponible sur : http://droit.francophonie.org/doc/html/znao/doc/dcum/ 2003dfznaodcum12.html.

76 - Articles 19, 20 et 21 du règlement $n^{\circ}$ 3/2002/CM/UEMOA. Pour une étude approfondie des procédures devant la Commission, voir COULIBALY (A.-S.), op. cit. (Note 34).

77 - Le principe est posé et explicité à l'article 16 du règlement n 3/2002/CM/UEMOA.

78 - Article 5 paragraphe 5.2 de la directive $n^{\circ}$ 02/2002/CM/UEMOA.

79 - Avis précité, op cit, P. 163.

80 - Il est à noter en effet que les sanctions pécuniaires que peut infliger la commission, notamment les amendes, n'ont pas de caractère pénal.

81 - Pour plus d'information, voir : Ayawa Améhia TSKADI, «De la compétence exclusive de l’Union en droit de la concurrence dans l'espace UEMOA », Revue droit uniforme africain, Actualité trimestrielle de droit et de jurisprudence $\mathrm{N}^{\circ}$ 005, 2011, PP. 9-12. Voir aussi en droit comparé la convention de l'UEAC, art 8, Al 2.

82 - La même solution existe en droit de l'union européenne et a une portée plus large que le droit de la concurrence, puisqu'elle s'applique aux différents règlements qui prévoient des sanctions.

83 - P. Meunier, «La Cour de justice des communautés européennes et l'applicabilité des règles de concurrence du traité CECA », RTD eur, 1996, P. 243 ; CJCE, 6 avril 1962, aff, 13/61, Rec., P. 97.

84 - Règlement $n^{\circ} 1 / 2003$ en droit européen préc. Communication de la commission sur la coopération entre la commission et les juridictions nationales pour l'application des articles 81 et 82 du traité CE.

85 - Règlement n¹/2003 précité, art, 16.

86 - L'Acte additionnel $\mathrm{n}{ }^{\circ} \mathrm{A} / \mathrm{SA} 1 / 06 / 08$ portant adoption des Règles Communautaires de la Concurrence et de leurs modalités d'application au sein de la CEDEAO;

L'Acte Additionnel $\mathrm{n}^{\circ} \mathrm{A} / \mathrm{SA} / \mathrm{SA}$. 2/06/08 Portant création et attributions et fonctionnement de l'Autorité Régionale de la Concurrence (ARC) de la CEDEAO. Le Cadre Régional de Politique de la Concurrence. 
87 - Pour cette information concernant le projet d'harmonisation dans le domaine du droit de la concurrence, voir notamment : - Philippe TIGER, «Le droit des affaires en Afrique (OHADA) », Presses universitaires de France, 1 janv. 1999, p. 29.

88 - Sur la compatibilité entre l'UEMOA et l'OHADA, voir notamment : DEMBA Boubacar BA, « Le problème de la compatibilité entre l'UEMOA et l'OHADA », in Centre d'études européennes et de l'intégration (CEEI), «Actes du colloque de Ouagadougou des 16 et 17 décembre 1999 portant sur le thème «La libéralisation de l'économie dans le cadre de l'intégration régionale : le cas de l’UEMOA», p. 157-195.

89 - Voir en ce sens, DEMBA Boubacar BA, «Le problème de la compatibilité entre l'UEMOA et l'OHADA », op. cit., p. 183-185.

90_ COULIBALY abousaïb, «le droit de la concurrence de l'union économique et monétaire ouest africaine », revue burkinabé de droit, $n^{\circ}$ 43-44, 1er et 2ème semestres, Ouagadougou 2003, p. 25. 